\title{
Die Siegesstele des Amasis
}

Summary: Republication of the 'Elephantine stela' of Amasis, formerly Cairo $13 / 6 / 24 / 1$, now in the Nubian Museum, Aswan. The inscription, in the Königsnovelle tradition, reports two episodes of the war between Apries and Amasis. The first part, dated to year 1 of Amasis, describes the counter-attack of Apries, with the help of Greek mercenaries, against the usurper Amasis (Oct./ Nov. 570). Contrary to earlier assumptions, Apries' fleet did not start from an 'island' $(j w)$ but rather traveled down the Canopic arm $\left({ }^{c_{n}} n\right)$ of the Nile. The attackers were defeated by Amasis' troops at Jmiw. The second part concerns repulsing an invasion by Babylonian ('Asiatic') troops trying, in year 4 (March 567), to reinstall Apries as king, who was killed in the course of the battle.

Keywords: $26^{\text {th }}$ dynasty - Amasis - Apries - Babylonian invasion - civil war - Cyprus

DOI 10.1515/zaes-2014-0011

Die Siegesstele des Amasis (Kairo TN 13/6/24/1; heute im Nubischen Museum Assuan im Garten aufgestellt) ist irgendwann vor 1880 ins Museum Kairo gebracht worden'; vorher war sie in Kairo in einem Palais als Schwelle verbaut ${ }^{2}$. Obwohl spätestens seit Daressys Publikation klar war, wie wichtig diese Inschrift für die Geschichte der 26. Dynastie ist und dass sie Geschehnisse berichtet, die auch bei Herodot und Diodor eine Rolle spielen, ist die Literatur darüber nicht allzu reichlich:

G. Daressy, „Stèle de l'an III d'Amasis“, RecTrav 22, 1900, 1-9 (bisher einzige Wiedergabe der gesamten Inschrift, allerdings mit zahllosen Fehlern; seine Übersetzung ist daher kaum von Nutzen).

1 Im Jahre 1900 war die Stele „plus de vingt ans“ im Museum Kairo, s. Daressy, RecTrav 22, 1900, 1.

2 PM IV, 71.

Prof. Dr. Karl Jansen-Winkeln: Freie Universität Berlin, FB Geschichts- und Kulturwissenschaften, Ägyptologisches Seminar, Altensteinstraße 33, 14195 Berlin,

E-Mail: jawinkel@zedat.fu-berlin.de
BAR IV, 509-12 (§ 996-1007: nur wenige Stellen).

G. Posener, „Notes sur la stèle de Naucratis“, ASAE 34, 1934, 143 (Zusammenstellung der verschiedenen Schreibungen von $m \check{s}^{c}$ „Armee“).

id., „Les douanes de la Méditerranée dans l'Égypte Saïte“, Revue de philologie, de littérature et d'histoire ancienne 21, 1947, 117-31 (bes. 128-30).

E. Edel, „Amasis und Nebukadrezar II.“, GM 29, 1978, 13-20 (wichtigste Behandlung der Inschrift seit Daressy; Übersetzung mehrerer Passagen und richtige Lesung und Erklärung der Daten; seither anerkannte Gleichsetzung der Invasion von „Asiaten“ im Jahr 4 des Amasis mit dem Angriff Nebukadrezzars in seinem 37. Jahr).

A. J. Spalinger, „The Civil War between Amasis and Apries and the Babylonian Attack against Egypt“, in: W. F. Reineke (Hg.), First International Congress of Egyptology, Acts, Berlin 1979, 593-604 (im Folgenden Spalinger, Civil War; Versuch einer historischen Einordnung der Vorgänge mit zahlreichen wertvollen Bemerkungen).

id., Aspects of the Military Documents of the Ancient Egyptians, New Haven, London 1982; Anführung einiger Stellen nach Daressys Text: p. 33; 40; 54 (12); 63 (4); 69 (4); 72; 77 (2); 80 (23); 82 (11); 108 (n. 40); 111-2; 113; 118 (n. 76).

A. Leahy, „The Earliest Dated Monument of Amasis and the End of the Reign of Apries“, JEA 74, 1988, 183-99 (Chronologie des Beginns der Regierung des Amasis; wichtigster Beitrag seit Edel; Kritik an dessen These, der Gegenangriff des Apries sei von Zypern ausgegangen).

A. Lloyd, Herodotus, Book II: Commentary 99-188. EPRO 43.3, Leiden 1988, 178-80.

P. der Manuelian, Living in the Past, London, New York 1994, 403 (nur Erwähnung); pl. 11-12.

I. Ladynin, „The Elephantine Stela of Amasis: Some Problems and Prospects of Study“, GM 211, 2006, 3156 (einige kühne Thesen, wie z. B. der babylonische Angriff habe sich gegen Apries gerichtet).

A. Cannavò, „La dominazione egiziana a Cipro e i rapporti fra Cipro e l'Egitto nel VI Sec. A.C.“, SCO 49, 2003, 137-158 (Besprechung und auszugsweise Übersetzung der Amasisstele auf p.141-8, weitgehend nach Edel). 
R. Gozzoli, The Writing of History in Ancient Egypt during the First Millennium BC (ca. 1070-180 BC). GHP 5, London 2006, 101-3 (Struktur des Textes der Stele).

A. Mehl, "The Relations between Egypt and Cyprus from Neo-Assyrian to Achaemenid Rule (7th-6th Cent. B. C.)”, in: D. Michaelides u. a. (Hgg.), Egypt and Cyprus in Antiquity, Oxford 2009, 60-66.

C. Balandier, „L'Égypte, Chypre et la route de Péluse à Gaza: approche micro-régionale des politiques stratégiques des derniers souverains égyptiens et achéménides (610-332 av. J.-C.)“, in: ibid., 78-96 (bes. 7980).

A. Blöbaum, „Denn ich bin ein König, der die Maat liebt““ Herrscherlegitimation im spätzeitlichen Ägypten. ÄgMon 4, Aachen 2006 (im folgenden: Blöbaum, Herrscherlegitimation); Erwähnungen der kollationierten Inschrift mit teilweise neuen Lesungen: 13 (Kol. 2-3; 4); 14 (Kol. 17-8); 34 (Kol. 2); 57 (Kol. 7); 65 (Kol. 2); 80 (Kol. 2); 83 (Kol. 2); 93 (Kol. 2); 135 (Kol. $17-$ 8); 164 (Kol. 2-3; 4; 17-8); 165 (Kol. 14); 204 (Kol. $10-$ 1); 224 (Kol. 2); 269 (Kol. 5-6); 271-2 (Kol. 16-7); 338.

Es ist unstrittig, dass die Siegesstele des Amasis eine der wichtigsten (der insgesamt recht wenigen) ägyptischen Quellen zur Geschichte der 26. Dynastie ist. Dennoch ist sie nur in einer sehr alten Edition (von 1900) bekannt, die zudem völlig unzureichend ist und zahllose sinnentstellende Fehler enthält. Da die Stele zunächst im $\mathrm{Mu}$ seum Kairo (bis etwa 1990 in R 24) relativ bequem zugänglich war und heute im Museumsgarten von Assuan offen aufgestellt ist, kann das nur daran liegen, dass ihre Lektüre sehr problematisch ist. Allerdings dürfte diese Stele, deren Zustand oft beklagt worden ist, paradoxerweise eine der wenigen ägyptischen Königsstelen sein, die vollständig erhalten sind. Tatsächlich zeigt es sich bei näherem Hinsehen, dass kein einziges Schriftzeichen wirklich vollständig verschwunden ist. Warum dennoch ihre Entzifferung so schwierig ist, hat Daressy treffend formuliert: „La surface en est usée par endroits, elle n'est plus polie; la lecture des inscriptions est des plus difficiles, les signes, peu profondément gravés, se perdent dans les inégalités du granit et n'ont laissé que des silhouettes sans détails“3. Auch Breasted hat betont, wie schwierig die Stele zu lesen ist: „The stone is much worn, and so illegible that sometimes not a single sign is certain for half a line or more“, und daraus folge: "a consecutive translation is totally impossible" ${ }^{4}$. Das ist allerdings stark übertrieben.
Die zweite Schwierigkeit, die das Verständnis der Inschrift (und damit auch ihre Entzifferung an vielen Stellen) behindert, ist ihre Orthographie; auch dies war schon von Daressy angesprochen worden. Es ist zwar nicht eine eigentlich „alphabetische“ Orthographie, wie das besonders in der Zeit Psametiks II. häufiger war, aber es werden viel weniger Determinative als üblich geschrieben. Es gibt z. B. im ganzen Text nur eine einzige Buchrolle $^{5}$ und keine Pluralstriche. Einige Beispiele für determinativlose Wörter: $s h r$ „Rat, Plan“ (Kol. 2, 2x); hiw „Umgebung“ (Kol. 3; 9); ph (alphabetisch geschrieben) „erreichen“ (Kol. 4; 11); sh(wj) „versammeln“ (Kol. 4); $m^{c} r$ „erfolgreich sein“ (Z. 5); sbj „rebellieren“ (Z. 5; 14); $d r$ „vertreiben“ (Z. 6); smsw „Pferde“ (Kol. 8); dismw „Rekruten“ (Kol. 8; 10); qn „Schaden, Unheil“ (Kol. 9; 15); $n d s$ „klein“ (Kol. 9); wr(ry)t „Streitwagen“ (Kol. 11); $m^{c} b 3$ „Speer“ (Kol. 11); zhn „suchen“ (Kol. 13); wdn „Opfer“ (Kol. 13); hpj „gehen“ (Kol. 14); nhm ,jubeln“ (Kol. 11; 16); $s n d m$ „sitzen“ (Kol. 17) und andere mehr. Dadurch ist die Abtrennung der Wörter in vielen Fällen nicht eindeutig markiert, und weil ganz überwiegend Lautzeichen gebraucht werden und deren Konturen oft undeutlich sind, steigen die Möglichkeiten ihrer Kombination und ebenso die Möglichkeiten abenteuerlicher Missdeutungen.

Die Hieroglyphen selbst sind zwar an den wenigen Stellen, die nahezu unbeschädigt sind, recht deutlich, aber ihre Erkennbarkeit ist doch ganz unterschiedlich ausgeprägt:

Besonders klar ist das $n$ mit sehr stark ausgeprägten „Zacken“ (meist vier oder drei). Die leichte Erkennbarkeit dieses häufigen Buchstabens, selbst bei starker Beschädigung, ist eine der größten Hilfen bei der Entzifferung dieses Textes. Gut erkennbar ist meistens auch die Negation, weil sie in der Mitte stark erhöht ist. Dagegen sind die Vogel-Hieroglyphen (besonders $m, w$ und 3) sich oft sehr ähnlich. Kleinere detailreiche Zeichen wie z. B. $\underline{d} r$ oder $\mathrm{km}$ werden nur grob eingetieft; das $\underline{d} r$ ist eigentlich nur ein kleines Rechteck. Weitere Beispiele sind das (mutmaßliche) $d d$ oben in Kol. 13 und das stp in Kol. 1 (stp ntrw). Besonders variabel ist die $f$-Schlange, von sehr stark gewellt bis nahezu waagerecht.

Während der Vorbereitung des vierten Teils meiner „Inschriften der Spätzeit“ (26. Dynastie) war es erforderlich, sich auch mit der Stele des Amasis ausführlich zu beschäftigen. Zu der Zeit, als die Stele noch im Museum Kairo bequem zugänglich war und verschiedene Beleuchtungen gestattete, hatte ich es leider versäumt, sie eingehend $\mathrm{zu}$ kollationieren. An ihrem neuen Aufstellungsort

5 In Kol. 14, und auch die ist unsicher. 


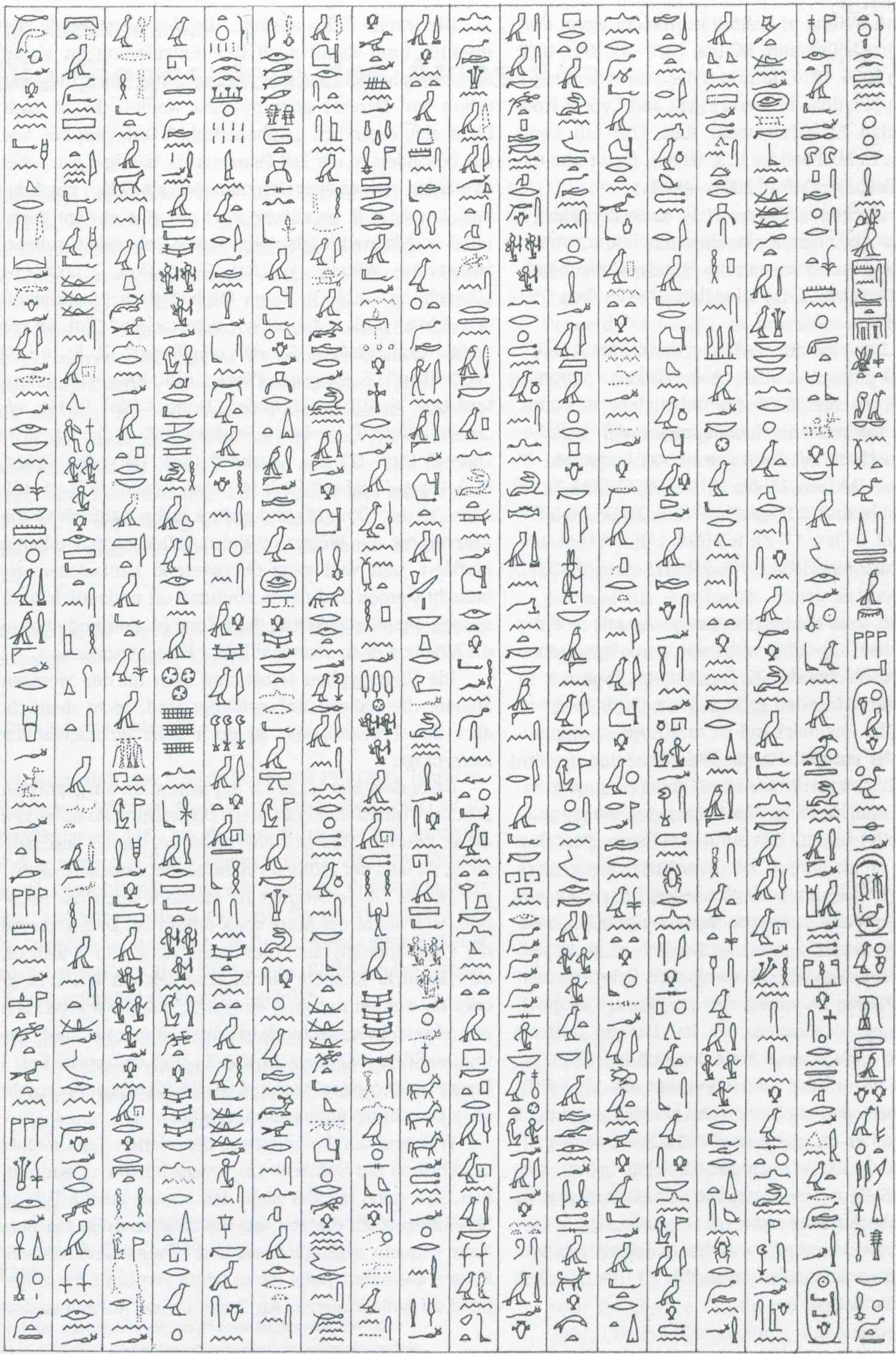

Abb. 1: Textfeld der Siegesstele des Amasis, Handkopie. 
im Museumsgarten von Assuan haben G. Vittmann und L. Borrmann eine Reihe von Fotos für mich gemacht, wofür ich ihnen herzlich danke, und ich habe die Stele dort auch selbst kollationiert. Die große Abhängigkeit vom Einfallswinkel der Sonnenstrahlen würde eigentlich eine Reihe von Besuchen erfordern, vermutlich am besten $\mathrm{zu}$ verschiedenen Jahreszeiten. Allerdings führt in den meisten Fällen das genaue Studium stark vergrößerter Fotos wohl weiter als die Autopsie der Stele. In jedem Fall ist es klar (und es geht auch aus den Anmerkungen zur Übersetzung hervor), dass es in meiner Abschrift noch viele Unsicherheiten gibt. Der hier neu publizierte Text ist eine (allerdings halbwegs maßstäbliche) Handkopie (Abb.1), kein Faksimile! Ich habe davon abgesehen, beschädigte Zeichen oder Passagen zu schraffieren; wegen der Menge der Schäden würde das nur die Lektüre erschweren. Besonders stark beschädigte oder unklare Zeichen(teile) sind gepunktet worden (es sind also keine Ergänzungen!). Es haben sich aber insgesamt so viele (auch sichere) Verbesserungen gegenüber Daressys Abschrift ergeben, dass eine neue Publikation (nach mehr als 110 Jahren) wohl gerechtfertigt ist und hoffentlich zu weiteren Korrekturen anregt.

Noch mehr Unsicherheiten als die Abschrift enthält die Übersetzung (s.o.), die zudem noch zahlreiche Lücken hat. Aber auch hier dürften die Struktur des Textes und der Sinn der meisten Passagen einigermaßen klar werden. Außerdem sind gerade in einigen für das historische Verständnis besonders wichtigen Abschnitten deutliche Verbesserungen möglich.

Ein großes Problem ist die fotografische Dokumentation: Viele der mir zur Verfügung stehenden Fotos von Ausschnitten der Stele sind bei der Entzifferung der einzelnen Zeichen überaus nützlich gewesen, wenn man sie am Bildschirm stark vergrößert. Um aber die zeichnerische Wiedergabe der Inschrift anhand gedruckter Fotos wirklich kontrollieren zu können, wären wohl professionell gemachte Aufnahmen (unter idealen Lichtbedingungen bzw. bei Nacht gut ausgeleuchtet) erforderlich. Es ist zu hoffen, dass bald eine neue Gesamtedition mit Faksimile und guten Fotos möglich sein wird ${ }^{6}$.

Der Aufbau des Textes ist deutlich, er ist in $12 \mathrm{Ab}$ schnitte ${ }^{7}$ gegliedert.

\section{Übersetzung}

\section{Datum (Jahr 1) und Königstitulatur}

1 Jahr 1, 2. šmw unter der Majestät des Horus $S m n-m)^{3} t$, des Königs von Ober- und Unterägypten „Die beiden Herrinnen“ Z3-Njtt Spd-tiwj, „Goldhorus“ Stp-ntrw Hnm-jb-R , des leiblichen Sohnes des Re Jch-msjw Z3-Njtt, geliebt von Chnum, dem Herrn des Kataraktengebiets und Hathor zu Gast in Biga ${ }^{(1)}$, dem alles Leben, Dauer und Glück gegeben ist ewig wie Re.

\section{Eulogie des Königs}

2 Der vollkommene Gott, der mit seinem Arm handelt ${ }^{(2)}$, von großer Kraft, mit trefflichem Plan, der vortreffliche König, von aufrichtiger Art ${ }^{(3)}$, von dessen Anblick jedermann lebt, wie (beim Anblick von) Re, der im Horizont aufgeht.

\section{Bericht, 1. Teil: Der König im Palast}

Seine Majestät war in seinem Palast von Sais, indem er sich um die Belange des ganzen Landes kümmerte. Da kam man ${ }^{(4)}$, um seiner Majestät zu sagen:

\section{Meldung an den König: Angriff der Söldner des Apries}

„Der Apries, 3 es befahren für ihn Seeschiffe, gefüllt mit zahllosen Griechen ${ }^{(5)}$, den ${ }^{c} n w$-Nilarm ${ }^{(6)}$. Ihre Herzen sind zuversichtlich, indem (sie sagen): ,(Es) ist in unseren Händen; es gibt niemanden, der vor ihm [Apries] standzuhalten gedenkt. ${ }^{(7)}$ Es ist ihnen eng (selbst) in der Weite ${ }^{(8)}$, es gibt keinen Gott, dem ihr bevorzugter Aufenthalt gemeldet wird 4 beim Verzehren seines Unterhalts ${ }^{\left({ }^{9}\right)}$. Sie wollen ganz Ägypten einnehmen, sie werden Sht-mfk3t erreichen ${ }^{(\mathbf{1 0})}$; es fliehen vor ihnen diejenigen, die dir ergeben sind.“

\section{Bericht, 2. Teil: Der König ruft seine Berater zu sich}

Seine Majestät veranlasste, dass man die Freunde des Königs und die Kriegsknechte ${ }^{(11)}$ versammelte, um sie hören zu lassen, was geschehen solle.
6 Eine solche Edition wird von A. Blöbaum vorbereitet.

7 Wobei die Einleitungen der Reden (z. B. $\underline{d} d$.jn $h m . f$ ) mit zu den Reden gezogen worden sind, obwohl sie strenggenommen ein Teil des „Berichts“ sind. 


\section{Ansprache des Königs: Anfeuerung zum Kampf}

Seine Majestät sagte 5 diese Ansprache $\left(m d t-r^{\prime}\right)$ : „Was dies(e Sache) betrifft ${ }^{(12)}$, so hört:

Was den Erfolg(reichen?) angeht (so gilt): Einer, der tapfer kämpft, ist der, der für seine Heimat kämpft ${ }^{(13)}$. Sein Herz ist kampfbereit, <um> sich seine Angehörigen wohlbehalten sein $\mathrm{zu}$ lassen ${ }^{(14)}$. Denkt daran, was geschehen soll: Es lohnt sich nicht, dass man all die herbeibringt, die sich dagegen sträuben ${ }^{(15)}$. Es steht euch dort kein Gott gegenüber ${ }^{(16)}, 6$ es sind keine Stark(armig)en im Kampf gegen euch. Ein ... ${ }^{(17)}$ ist (dagegen) derjenige, der seinem ... ergeben ist ${ }^{(18)}$, sein ... ergreift(?) eure(?) ... ${ }^{(19)}$ Wer im Schutze seines Gottes kämpft, hat den (je) einer vertrieben, der sich (nur) auf Gewalt stützt ${ }^{(20)}$ ? Man fürchtet sich vor dem Großen, wenn er kampfbereit ist. Wer ist es denn (sonst), der veranlasst, 7 dass wirklich etwas herauskommt ${ }^{(21)}$ ?“

\section{Antwort der Höflinge: Zustimmung}

Sie sagten zu seiner Majestät: „Die Maat ist die Führerin deines Herzens, o Herrscher, unser Herr! Der Ratschluss Gottes ist dir zu eigen ( $j w n . k s h r n n t r$ ), wenn du die Diener und alle Deinigen siehst ${ }^{(22)}$. Der Wütige (dagegen) [= Apries], er hat gehandelt wie der Hund am Kadaver ${ }^{(23)}$.

8 Deine Pferde sind zahlreich, deine Bogentruppe, sie ist ohne Ende, die Leibgarde ${ }^{(24)}$, man kennt nicht ihre Zahl, es gibt keinen Mangel an ausgesuchten Rekruten ${ }^{(25)}$. Du bist ihr Herr!

Was den betrifft, der unsere Häuser (= Familien) schützt und die Nahrung von jedermann besorgt, wie wohlversorgt sind seine Mitbürger durch ihn, denn er ermutigt das Herz, 9 so dass es keinen gibt, der sagt ,hätte ich doch!' darunter ${ }^{(26)}$. Wer ist es (denn), der sie abwehrt, weil sie (doch) zahlreich sind gegen unser Land? ${ }^{(27)}$ Deine Majestät ist es!

Es gibt(?) keinen ${ }^{(28)}$, der nicht für es [das Land] kämpft. Wer (nur) für sich handelt, ist gering; ein Starker ist (dagegen) der, der unter deinem Schirm ist. Es gibt keine Not $<$ für $>$ einen, der in deinem Haus ist ${ }^{(29)}$.

Siehe, der Aufenthalt (?) in der Nähe deiner Diener ${ }^{(30)}$... 10 der Rekruten, denn ... ${ }^{(31)}$ die Füße des Fliehenden(?) standhaft zu machen. ${ }^{(32) ، ~}$

\section{Abschließende Aufforderung des Königs}

Da sagte seine Majestät: „Ihr sollt kämpfen! Jeder einzelne möge den ihm gegenüber befindlichen (Feind) tö$\operatorname{ten}^{(33) ! “}$

\section{Bericht, 3. Teil: Vorbereitung zum Kampf, Schlacht, Dankesopfer}

Dann war seine Majestät dabei, sein Heer zu inspizieren und seine Pferde zu sichten(? $)^{(34)}$, die er ausgewählt hatte ${ }^{(35)}$ unter der Majestät des (36). Seine Majestät stellte sich 11 auf seinen Streitwagen, nachdem er seinen Speer ${ }^{(37)}$ ergriffen hatte wie ein Gott und der Bogen von Silber(?) ${ }^{(38)}$ in seiner Hand war mit der Nennung seines Namens auf Befehl der Neith ${ }^{(39)}$. Er erreichte $J m 3$ iw (Kom el-Hisn), indem das Heer in Jubel war und Jauchzen auf allen Wegen (herrschte). Die Schlachtreihe(?) wurde aufgestellt ${ }^{(40)}$, bevor ihre gute Stimmung sich verdüstern konnte $^{(41)}$... 12 dort, indem gekämpft wurde (o.ä.). Sie wurden aufgestellt, indem ihr Platz war an ${ }^{(42)}$... um(??) sich nicht von ihm zu trennen(??) ${ }^{(43)}$.

Dann kämpfte seine Majestät wie ein Löwe, um sich ein großes Gemetzel anzurichten unter ihnen, ohne dass man ihre Zahl kannte. Viele Seeschiffe waren gekentert, und die Kämpfer waren ins Wasser gefallen. Sie wurden treiben gesehen, $\mathbf{1 3}$ wie es Fische tun. Die ganze Zeit dauerte an, indem es kein Ablassen vom Kämpfen gab $^{(44)}$. Die Sonne ging unter ${ }^{(45)}$, indem sein [des Königs] Herz froh war. Seine Majestät veranlasste, dass man den ${ }^{n} n$ Nilarm abriegelte auf all seinen Seiten ${ }^{(46)}$.

Zahllos waren die, die <zum> Gott gingen ${ }^{(47)}$, der vernachlässigt worden war und das Opfer gesucht hatte ${ }^{(48)}$, indem sie beladen waren und kein Ende war an dem, was sie zu tun hatten.

\section{Datum (Jahr 4), Meldung an den König: Invasion der Asiaten}

14 Jahr 4, 3. 3ht, Tag 8; man kam, um ${ }^{(49)}$ seiner Majestät zu sagen: „Die Asiaten haben sich in ihrer Überheblichkeit empört, und sie ziehen heran auf dem Horusweg. Es sind Tausende dort und greifen das Land an: Sie bedecken jeden Weg, und die zu Schiff kommen, sie segeln, indem ihre Herzen planen, 15 unser Land umzustürzen.“ 


\section{Schwur des Königs, die Feinde zu vernichten}

Seine Majestät sagte zu seiner Armee: „So wahr ich lebe und $\operatorname{Re}<$ mich $>$ liebt, und so wahr meine Nase jugendfrisch im Leben ist ${ }^{(50)}$ : Wer Schaden anrichtet in den Städten und Gauen, (meine) Majestät wird nicht aufhören, ein Gemetzel anzurichten. Die Armee meiner Majestät ist zahlreich auf allen Wegen. Nicht zulassen wird der Tag 16 ... eure Arme unter ihnen ${ }^{(51)}$.

Der Frevler, der keinen Namen hat, wird auf (?) dem Schlachtfeld(?) $\operatorname{sein}^{(52)}$... als einer, der sich nach vorn begibt"“(53).

\section{Bericht, 4. Teil: König vor Gott, Unwetter, Schlacht, Dank an die Götter}

Seine Majestät stand beim Kultbild des Gottes ${ }^{(54)}$, während seine Armee bis zum Himmel jubelte ${ }^{(55)}$. Der Gott trachtete danach ${ }^{(56)}$, ihm zu öffnen(??) 17 den Himmel $^{(57)}$ in einem Hagelsturm, so dass ihre Schiffe kenterten. Die Soldaten stiegen herab(?) ${ }^{(58)}$, indem sie Gemetzel unter ihnen anrichteten, (sie) vernichteten und sie erbeuteten. Nachdem(?) sich seine Majestät in seiner Kajüte niedergelassen hatte ${ }^{(59)}$, erblickte er den ,Überheblichen', der gefallen war durch das ${ }^{(\mathbf{6 0})}$, was er getan hatte, 18 indem seine Leiche ${ }^{(61)}$ im Wasser trieb. Dann bestattete er den Überheblichen, indem er alles für ihn tat, was für jeden rechtmäßigen König zu tun ist ${ }^{(62)}$. Dann begab sich seine Majestät zu seinem Palast, nachdem er den Abscheu der Götter entfernt hatte ${ }^{(63)}$ und nachdem er dauerhaft ein sehr großes Gottesopfer gestiftet hatte für die Götter von Ober- und Unterägypten, damit er mit Leben beschenkt sei wie Re ewig.

\section{Anmerkungen}

(1) Falls, wie zu erwarten, hier die Insel Biga gemeint ist, müsste das seltsame Zeichen neben dem Geier, das keiner gängigen Hieroglyphe entspricht, $s n$ zu lesen sein.

(2) Unter dem Auge ist wohl ein (überflüssiges) $t \mathrm{zu}$ lesen; für ein bloßes Loch im Stein ist die Kontur zu klar.

(3) Das Zeichen nach $m t j$ bjt ist sehr unsicher, und es wäre als Determinativ für bjt „Charakter“ ja auch unüblich. Blöbaum (Herrscherlegitimation, p. 93) liest $m$ tr $b j 3 . t=f$, aber der Abstand zwischen bjt und $c_{n h} h r n b$ ist deutlich zu groß für ein $f$, das auch nicht den Spuren entspricht. Außerdem wäre ein Suffix in dieser Konstruktion ungrammatisch.
(4) Die „Beinchen“ neben $j j$ sind kaum zu erkennen, vielleicht steht auch nichts an der Stelle.

(5) Wörtlich: „deren Grenze man nicht kennt“, vgl. Wb V, 588,3; 10. Zum Ausdruck hỉw-nbwt s. J. Quack, „Das Problem der H3w-nb.wt“, in: R. Rollinger (ed.), Getrennte Wege? Kommunikation, Raum und Wahrnehmung in der Alten Welt, Frankfurt 2007, 331-62.

Blöbaum (Herrscherlegitimation, p.13, mit n. 101) schlägt eine gänzlich andere Lesung vor: statt kbnwt mh.tj $m$ his-nbwt (so schon De Meulenaere in: C. Vandersleyen, Les guerres d'Amosis, Brüssel 1971, 144 und Edel, GM 29,

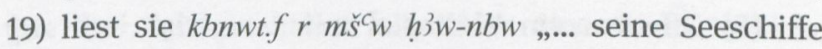
hin zu den Truppen der Griechen“. Das halte ich für vollkommen ausgeschlossen: Das von De Meulenaere und Edel $m h$ gelesene Zeichen hat zwar eine etwas merkwürdige (geknickte) Form, es ist aber sehr viel eher ein $m h$ als ein $f$, und darunter steht ein $t$, ganz sicher kein $r$. Die Lesung $t j$ halte ich gleichfalls für sicher.

(6) Das Zeichen, das bisher $j w$ „Insel“ gelesen wurde, ist zum einen ungewöhnlich dick und hat zweitens definitiv eine Innenzeichnung, und zwar ziemlich sicher das Auge mit dem Lidstrich darunter (๕). Darunter steht vermutlich das (hier etwas kurvig geschriebene) Determinativ $\square$ (so auch Blöbaum, Herrscherlegitimation, p. 13, n. 100), nicht das Suffix f. Dieselbe Schreibung kommt noch einmal in Kol. 13 vor. Es dürfte also $c_{n}(w) \mathrm{zu}$ lesen sein, und dies ist auf der Naukratisstele Nektanebos' I. der Name des Nilarms von Kanopos, s. u., Kommentar (B).

Die Konstruktion des Satzes ist erläuterungsbedürftig. Edel, GM 29, 19 übersetzte: „die Insel ... setzt für ihn Seeschiffe über“. Dann wäre eine Ortsbezeichnung („die Insel“) das Subjekt zum Verb hnj „rudern, fahren“8 (was in Edels Übersetzung etwas verdeckt wird) und die Schiffe das Objekt. Eine solche Formulierung (ein Ort „fährt“ Schiffe) dürfte schwerlich möglich sein. Es scheint sinnvoller, die Schiffe zum Subjekt zu machen, denn „ein Gewässer befahren“ ist für das Verb $\underline{h n j}$ belegt (Wb III, 374,25-26). Dann wäre allerdings die Wortstellung nicht normal, weil das Objekt dem Subjekt voranginge. Da es sich aber um ein kurzes Objekt und eine sehr langes Subjekt handelt (kbnwt mh.tj $m$ hỉw-nbwt $n$ rh.tw drw.sn), wäre eine Nachstellung wohl möglich (vgl. Gardiner, EG, $\S 507.3$ ); vergleichbare Konstruktionen sind auch im Mittelägyptischen der Spätzeit bezeugt (s. K. Jansen-Winkeln, Spätmittelägyptische Grammatik. ÄAT 34, Wiesba-

8 Die Umschrift hnt.n.f bei Leahy, JEA 74, 1988, 193 ist wohl ein Versehen. Die Lesung des Zeichens für hnj (sign-list D 33) ist jedenfalls gesichert. 
den 1996, §608). Die feminine Form mh.tj bei pluralischem Bezugswort entspricht übrigens nicht klassischem Standard, ist aber im Spätmittelägyptischen durchaus üblich, vgl. etwa D. Kurth, Einführung ins Ptolemäische, Teil 2, Hützel 2008, 728 (oben); es könnte sich umgekehrt auch um einen Archaismus nach Vorbild des Alten Reichs handeln.

(7) $\mathrm{Zu} c^{c} h^{c} m h 3 w . f$ s. Wb II, 477,7. Das Zitat der Söldner wird durch $h r<\underline{d} d d>$ eingeleitet, s. Gardiner, EG §321; Jansen-Winkeln, Spätmittelägyptische Grammatik, §619; 648.

(8) Offenbar ein Wortspiel mit hns „eng“ und wsh „weit“; vermutlich eine Umschreibung für so etwas wie „sie machen sich überall breit“.

(9) $\mathrm{Zu} \operatorname{sh} 3$, $n \mathrm{~s}$. Wb IV, 233,26; gemeint ist wohl, dass man den Gott (bzw. die Priester) nicht um Erlaubnis fragte, wenn sich die Truppen Besitztümer der Tempel aneigneten (vgl. im einzelnen unten den Kommentar [H]). Die Phrase zu Beginn von Kol. 4 verstehe ich als $m$ wnm ${ }^{n h}$. $f$ (Wb I, 205,8). Zur Schreibung ${ }^{n} n \check{s}$ s. Wb I, 193, aber die Wiedergabe von $h$ durch $\check{s}$ ist in der Spätzeit allgemein nicht sehr selten und kommt auch auf Königsstelen der 26. Dynastie vor, z. B. auf der Shellal-Stele Psametiks II, s. Der Manuelian, Living in the Past, 341; pl.16, Z. 5 (hnš $>$ šnš).

(10) Zu Sht-Mfk(3t) s. Gauthier, DG V, 53; Montet, Géographie, I, 62; Spalinger, Civil War, 602-3 (15). Aufgrund des Elements $m f k 3 t$ nimmt man allgemein an (vgl. auch Leahy, JEA 74, 1988, 190), dass damit Terenuthis/Kom Abu Billu gemeint ist.

(11) Die Bezeichnung $h m w n r^{\prime}-c^{-} h t$ scheint sonst nicht belegt zu sein. Ob damit speziell die Söldner(führer) gemeint sind?

(12) Das $r$ von $j r$ ist nur hier vollständig ausgehoben und etwas unregelmäßig geformt. Es kommt aber kaum eine andere Lesung in Betracht (sicher nicht $f$ wie bei Daressy).

(13) Mit $w(w)$ „Bezirk“ kann in diesem Zusammenhang nur so etwas wie „Heimat“ gemeint sein. Allerdings kann 'h? hr sowohl „kämpfen für“ etwas wie auch „an“ einem Ort bedeuten (Wb I, 215, 10-11). „In seinem Bezirk, in seiner Heimat kämpfen" wäre natürlich auch sinnvoll. Syntaktisch liegt ein Nominalsatz mit direkter Nebeneinanderstellung (ohne Kopula) vor, wie es gerade in sentenziösen Formulierungen häufig ist, vgl. Jansen-Winkeln, Spätmittelägyptische Grammatik, §571; 706; id., Sentenzen und Maximen, Berlin 1999, 25-6.

(14) $\langle r\rangle$ sw 3 d n $f$. hnw.f. Auslassung der Präposition $r$ auch in Kol. $13(<r>3 w . s ; s ̌ m<r>n t r)$.

(15) Wörtlich „es gibt keinen Lohn, weil man herbeibringt ..... Zur Stellung des hr.s zwischen dem Partizip $s b j(w)$ und $n b$ vgl. Jansen-Winkeln, Spätmittelägyptische Grammatik, §421. Gemeint ist offenbar, dass man niemanden rekrutieren soll, der nicht willens ist zu kämpfen.

(16) Blöbaum (Herrscherlegitimation, p. 269) liest nțr jm $m$ hr.tn „der Gott ist da bei euch“, also ohne die Negation davor (die in der Tat nicht sicher, aber doch sehr wahrscheinlich ist). So wie ich den Satz verstehe, müsste mit ihm der Gedanke beginnen, dass der Gegner gottlos und auch nicht sonderlich kampfstark ist.

(17) Das erste Zeichen unter $c h 3$ r.tn könnte der $w r$ Vogel sein, darunter links ein $b$ und rechts davon vielleicht der $j b 3$-Spielstein. In den Inschriften des Harwa (BIFAO 30, 1931, 804; BIFAO 34, 1934, 154) wird damit das Wort $j b w$ „Zufluchtsstätte“ geschrieben. Es ließe sich daher hier so etwas wie „von großer Zufluchtsstätte“ = „einer, der einen bedeutenden Schutz hat, ist jemand, der auf dem Wasser seines [Gottes? Herrn?] ist“ vermuten, aber das ist schon mehr als unsicher.

(18) Die halbe Gruppe über dem . $f$ ist nicht zu erkennen. Es könnte sich um eine Bezeichnung des göttlichen oder menschlichen Herrn handeln, aber dem Zusammenhang nach wäre „auf dem Wasser seines Gottes“ wahrscheinlicher. Eine Lesung ntr. $f$ ist allerdings dem Raum und den Spuren nach nicht möglich.

(19) Alles ganz unsicher: Der Gruppe über $h r$ kann ich keinen Sinn abgewinnen, unter $m h($ ?) $m$ („,ergreifen“?) ist wohl dr.tn zu lesen, aber der Sinn ist fraglich.

(20) Bei $j n-j w$ kann es sich nur um die Fragepartikel handeln, und eine rhetorische Frage passt hier ja auch gut. Die Konstruktion, ein Fragesatz mit einer längeren Objektsphrase in Prolepse, ist aber zumindest ungewöhnlich.

(21) Der Satz $\left(<j n\right.$ ?> $m^{\prime}$ tr rdj prj (j)ht $m$ wn $\left.m^{3{ }^{3}}\right)$ scheint phraseologisch ausgesprochen dubios, obwohl prj im Sinne von "sich ergeben“ tatsächlich belegt ist (Wb I, 525,2).

Mit diesem Satz, wie auch immer zu verstehen, endet in jedem Fall die Ansprache des Amasis. Seine Argumentation, falls hier richtig verstanden, scheint folgendermaßen zu sein: Seine Leute werden tapfer kämpfen, da es um ihre Heimat geht, aber man soll nur wirklich Kampfeswillige heranziehen. Der Gegner hat keinen göttlichen Beistand und ist nicht besonders stark, man selbst hat dagegen die Unterstützung der Götter, und der eigene Führer ist gut vorbereitet.

(22) Ich lese (mit erheblichen Zweifeln) $t w(t) n b$, also das selbständig gebrauchte „neuägyptische“ Possessivpronomen, vgl. etwa J. Černý/S. Groll, A Late Egyptian Grammar, Rom 1975, 21 (v. a. Ex. 44: p3 swt nb ,alles seinige"). 
(23) Das soll wohl heißen: Amasis handelt wohlüberlegt, Apries dagegen raubgierig und ohne Plan. S. auch unten den Kommentar (E).

(24) $\mathrm{Zu} m h(w)-j b$ im Sinne von „Leibgarde“ o. ä. vgl. Wb II, 118,17-119,1; P. Wilson, A Ptolemaic Lexicon, Löwen 1997, 449-50. Es ist bemerkenswert, dass dieses (seltene und leicht missverständliche) Wort determiniert wird, während smsw „Pferde“ und $p d(t)$ „Bogentruppe“ keine Determinative haben.

(25) $n n$ wn g3w d3mw $n$ stp; zu giw mit folgendem direkten Genetiv s. Wb V, 152,13 und zu $n$ stp Wb IV, 339,11 .

(26) Das $j$ von $j m$ ist zweifelhaft, das Zeichen sieht je nach Lichteinfall recht unterschiedlich aus. Es könnte auch ein $w h m$ oder $\mathrm{nm}$ sein.

(27) Ich lese (mit großem Vorbehalt) jn-m gfn st $\underline{d r} r_{\breve{S}}$. sn $r$ t3.n. . kë könnte eine Umsetzung des neuägyptischen Frageworts " in eine „klassisch“ aussehende Form sein. Das Wort dahinter ist vielleicht ein Partizip von gnfl $g f n$,abweisen, abwehren“ (Wb V, 174, 9-11). Das ${ }^{(5)}$ ' hinter $d r$ ist wieder unsicher.

(28) Das wn (so auch Daressy) ist sehr unsicher.

(29) $j r j h r h^{c} f$ (wörtlich ,wegen seines Leibes“) $n d s(w)$, $n h t p w n t j \underline{h r}$ šwt.k; $n q n<n>n t j m$ pr.k.

(30) Die gleiche Phrase ist in Kol. 3 nach Wb I, 218,5 als „standhalten vor" übersetzt worden. Hier scheint allerdings ein „wer standhält vor deinen Dienern“ schlecht in den Zusammenhang zu passen, es ist ja offenbar von den eigenen Truppen die Rede. Die Lesung der beiden letzten Gruppen von Kol. 9 ist unsicher: unter dem $n n$ ist rechts vielleicht $b$ oder $\mathrm{nm}$ zu lesen, und der Vogel daneben könnte ein $w$ oder ein 3 sein. Auch die letzte Gruppe (unter $n$ ) ist unklar. Der Satz ist mir daher insgesamt noch unverständlich.

(31) Unter $h r-n t t$ (oder $h r n t j(w)$ ?) stehen ein $m$ und vermutlich ein $g$ oder ein $\underline{h} r$; ich kann nichts damit anfangen.

(32) smn tbwj $n$ wth (?). Das $w$ ist nicht zentral gestellt, rechts daneben sollte eigentlich noch ein Zeichen sein; ich kann aber nichts erkennen.

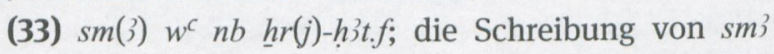
wäre sehr ungewöhnlich, zu der von $w^{c} n b$ s. Wb I, 276,7.

(34) Das Zeichen unter dem $h$ ist sehr beschädigt, aber es ist durchaus möglich, darin mit Daressy den Rest eines $f$ zu erkennen. Auch Edel (GM 29, 19: „seine Majestät musterte sein Heer und besichtigte seine Pferde“) liest offenbar $h f$.

(35) Eigentlich „(positiv) unterscheiden“, oft im Sinne von „erhöhen, auszeichnen“ (Wb V, 374); bei Pferden kann das nur die Auswahl der (für den Krieg) geeigneten Tiere bedeuten.
(36) Sic! Die Lesung des unteren Teils der Kolumne als nfrw. thn.n.f $h r h m n$ ist für jedes einzelne Zeichen ganz sicher; es ist weder mit Daressy $h m . f$ zu lesen noch mit Edel hr.sn („,bei ihnen“). Und ebenso sicher ist die Lesung der letzten Gruppe, wo mit $c h^{c} h m . f(h r$ wrryt.f) ein neuer Satz beginnt. Es ist evident, dass hier der Name des Königs, bei dem es sich nach Lage der Dinge nur um Apries handeln kann, einfach ausgelassen worden ist (s. dazu auch unten den Kommentar [E]).

(37) Aufgrund des Kontextes und der Stellung der Zeichen ist (mit Daressy) sicher $m^{c} b$ ' „Speer“ zu lesen. Die drei Zeichen haben allerdings eine ungewöhnliche Form, die eher an einen $m d w$-Stab erinnert. Da $m d w$ „10“ und $m d w$ „Stab“ lautlich zusammengefallen waren (Мнт), könnte man eine Ersetzung des Zeichens für 10 durch den Stab denken, so wie ja umgekehrt der Stab mit der „10“ geschrieben werden kann (Wb II, 184). Dann wäre allerdings die lautliche Identität nur genutzt worden, um ein neues Zeichen für „10“ zu gewinnen, das dann dreimal geschrieben wird, weil der Lautwert von „30“ dem von „Speer“ entspricht.

Es ist wohl einfacher anzunehmen, dass der Steinmetz die drei Zeichen für „10“ nur sehr nachlässig graviert hat.

(38) Edel (GM 29, 19) liest „Gold“, und das ist auch möglich. Darüber ist aber eine Art Kreis, was noch am ehesten zum $h \underline{d}$-Zeichen in der Goldhieroglyphe passen würde. Das Determinativ darunter ist ganz unsicher.

(39) $\mathrm{Zu} h r n \underline{d} r n . f \mathrm{~s}$. Wb II, 371,11-12. Das Verständnis der Gruppen darunter als $m w d t ~ N j t t$ scheint mir - trotz einiger Beschädigungen - sicher zu sein. Was Edel mit seinem ,in einem Augenblick (?) (erreichte er J3mw)“ gelesen hat, ist mir nicht klar. Zu weiteren Bemerkungen $\mathrm{zu}$ dieser Stelle s. den Kommentar (H).

(40) Die Lesung $t z$ ist wahrscheinlich, aber keineswegs sicher, die von $s k w$ sehr unsicher. Zu $t z s k w$ (falls so zu lesen ist) vgl. Wb IV, 314,6; V, 398,9. Allerdings dürfte dieser mit $t(3) z$ „knüpfen“ gebildete Ausdruck eher die Aufstellung zur Schlacht bezeichnen als den Beginn des Kampfes, wie auch die Belege des Wörterbuchs zeigen.

(41) Möglicherweise $n w h(3) s b i q . s n$ zu lesen. $\mathrm{Zu} w h$ „dunkel werden“ s. Wb I, 352,3-4; das Verb ist auch (wie hier) in metaphorischer Bedeutung belegt, s. H. von Deines/W. Westendorf, Wörterbuch der medizinischen Texte, I, Berlin 1961, 207. Zu sb3 $q$ im übertragenen Sinne („heiter stimmen, beglücken“) s. Wb IV, IV, 86,18-21 (dort auch die seit dem Neuen Reich häufige Schreibung $s q b)$. Syntaktisch ist der Satz wohl als temporaler Nebensatz zu verstehen, mit dem Verb in der Form $n$ s $d m t . f$ (dessen Endung $t$ im Mittelägyptisch der Spätzeit meist nicht ausgeschrieben wird, s. Jansen-Winkeln, Spätmit- 
telägyptische Grammatik, §113; D. Kurth, Einführung ins Ptolemäische, Teil 2, 2008, §152). Der Rest der Kolumne ist mir unverständlich.

(42) Ob von der Aufstellung des Heeres (jrj.tw.sn st.sn $m$...) die Rede ist? Die Gruppe nach $m$ wieder unklar.

(43) Nur als sehr gewagter Vorschlag: $r$ tm jwd $r f$. Dann müsste $j w$ für $r$ stehen (in diesem Text eher unwahrscheinlich), und die Lesung $\mathrm{tm}$ ist ohnehin ganz unsicher. Immerhin wäre aber dem Kontext (zwischen Ankunft des Heeres und dem Beginn des Kampfes) und den Wörtern st „Platz, Stelle“ und jwd „trennen“ nach eine Aussage über die Disposition der Truppe nicht ganz unwahrscheinlich.

(44) dd dj (?) phryt $<r>3 w . s n(n) 3 b w m$ ch 3 . Die Lesung der ersten Gruppe ist sehr unsicher, man erkennt eigentlich nur zwei unregelmäßige Rechtecke (vgl. oben).

(45) ${ }^{c} q r^{c}$ (s. Wb I, 230,8), so schon von Edel verstanden.

(46) Die Lesung des Verbs als $d n(j . t w)$ (und nicht $s n$ wie bei Daressy) ist sicher. Die Bedeutung „abdämmen“ (Wb V, 464,10-12) ist hier natürlich im militärischen Sinne zu nehmen: Zugang und Ausgang der Wasserstraße werden an allen Seiten verschlossen.

(47) $n r^{\prime}-{ }^{c} m \check{s} m(w)<r>n t r$, wörtlicher „es gab (dann) kein Ende an denen, die <zum> Gott gingen; die Lesung von $n r^{\prime}-c$ ist allerdings nicht sicher.

(48) ntr $m k h h$ j wn hr zhn wdn. Die Bedeutung „suchen“ ist zwar bei $z h n$ eher selten, aber sicher belegt, s. Wb III, 469, 3-4; R. Faulkner, The Ancient Egyptian Coffin Texts I, Warminster 1973, 256, n. 9 ( $z h n$ parallel zu hh hij).

(49) Die Schreibung jr wird ein Archaismus sein, der auch auf der Feldzugsstele Psametiks II. in derselben Formel vorkommt (Z. 5), ebenso in Z. 12 und auf der Stele des Apries in Memphis (Z. 3; 12), s. Der Manuelian, Living in the Past, pl. 16; 19.

(50) Ebenso schwört Pianchi, als er die schlecht gepflegten Pferde seines Gegners Nimlot entdeckt (Z. 65 der großen Pianchistele, s. Urk. III, 21); ganz ähnlich auch Thutmosis III. vor der Schlacht bei Megiddo (Urk. IV 651, 2-5).

(51) Man könnte so etwas erwarten wie „der Tag (des Kampfes) wird nicht gestatten, dass eure Arme (untätig sind) unter ihnen", aber die Spuren oben in Kol. 16 scheinen nicht dazu zu passen. Es ist auch nicht sicher, dass $h r w$ das Subjekt zu $r d j$ ist; die (viel seltenere) Konstruktion $n n r d j(. j) h r w$ (+ Pseudopartizip) wäre ebenfalls nicht auszuschließen. In Kol. 16 muss man auch nicht zwingend ${ }^{c}$.tn lesen; der „Arm“ könnte auch noch als Lautzeichen zum vorhergehenden Wort gehören.

(52) Von der Negation über $r n$ ist nur die linke Hälfte erhalten, eine andere Lesung kommt aber kaum in Frage.
Mit dem „namenlosen Frevler“ kann nur Apries gemeint sein. Man vergleiche auch Kol. 10, wo sein Namen ausgelassen wird. Nach $n r n$. $f$ folgt $m p t r$, danach $n b$ oder $k$. An sich könnte ptr eine Schreibung für prj „Schlachtfeld“ (Wb I, 532,1) sein, das in der Spätzeit häufig ptr geschrieben wird, s. Wilson, A Ptolemaic Lexikon, 381. Das würde gut in den Zusammenhang passen, allerdings sollte man für „auf dem Schlachtfeld“ eigentlich $h r p(t) r$ erwarten. Aber in Kol. 11 wird für „auf allen Wegen“ auch $m$ w3wt nb gebraucht (wo man ebenso $h r$ erwarten sollte). Die folgenden beiden Worte kann ich nicht erklären.

(53) Sehr fraglich: $m$ dj $\{t\} s w m$-hntj?

(54) $c^{c} h m . f h r c h m n n t r$, d. h. $n n t \underline{r}$ ist ${ }^{c} h^{c} h m . f h r c_{s ̌ m}$ vorangestellt, vgl. Z. 7, wo $n t r$ vor $\operatorname{shr} n$ steht. Diese Passage von $c h 3$ h $h m . f$ bis sndm $m$ snt in Z. 17 auch bei Blöbaum, Herrscherlegitimation, 271-2.

(55) Es ist $m \check{s}^{c}$.f $h r n h m r h r t$ zu lesen, nicht $m \check{s}^{c}(h r)$ $n h m$ hr pt wie bei Blöbaum, loc. cit.

(56) Blöbaum, loc. cit., liest und möchte es noch zum vorherigen Satz ziehen: „... jubelte zum Himmel der Ewigkeit“. „Himmel der Ewigkeit“ wäre aber eine sehr ungewöhnliche Wendung, und die Schreibung des $n$ darunter passt auch nicht recht. Die Lesung der Sonnenscheibe zwischen den beiden $h$ ist zwar nicht ausgeschlossen, aber keineswegs zwingend. Es handelt sich wohl eher um das Verb $h h j$ in der sdm.n.f-Form.

(57) Blöbaum, loc. cit., 271-2, liest unter ntrr „öffnen“. Das ist an sich eine gute Idee; allerdings wäre das $w b$ j-Zeichen dann sehr entstellt, und die Buchrolle zumindest nicht sicher (es könnte sich auch um das Schilfblatt handeln). Vor allem aber kann ich beim besten Willen den Ba-Vogel nicht erkennen. Am Beginn von Kol. 17 liest auch Blöbaum pt, aber die Anordnung der Zeichen (Lautzeichen unter Ideogramm) ist natürlich merkwürdig.

(58) Oder: „kamen herbei“. Der Vogel ist ziemlich sicher ein 3, rechts davon könnte man mit etwas Phantasie noch Reste des Unterteils von $\square$ erkennen, der obere Teil ist ganz zerstört. Die Beinchen (D 54) darunter sind allerdings sicher. Blöbaums Lesung n. 656) halte ich für ausgeschlossen. Zudem wird $c h 3$ auf dieser Stele immer ohne lautliches Komplement geschrieben (Kol. 5 [2x]; Kol. 9; 10; 12 [3x]; Kol. 13).

(59) Die Gruppe unter $m$ ist ganz unklar, $-\varangle$ ist mehr geraten als gelesen, aber auch Blöbaum und Edel (GM $29,17)$ lesen offenbar so. Zur Konstruktion vgl. Gardiner, EG, §327, aber satzeinleitend wäre sie in dieser Form dennoch ungewöhnlich. Auch die Gruppe darunter ( $h m . f)$ ist recht unsicher; $m$ - $h t ~ h m . f j w j . f$ wie bei Blöbaum halte ich aber der Lesung nach (und grammatisch) für ausgeschlossen. 

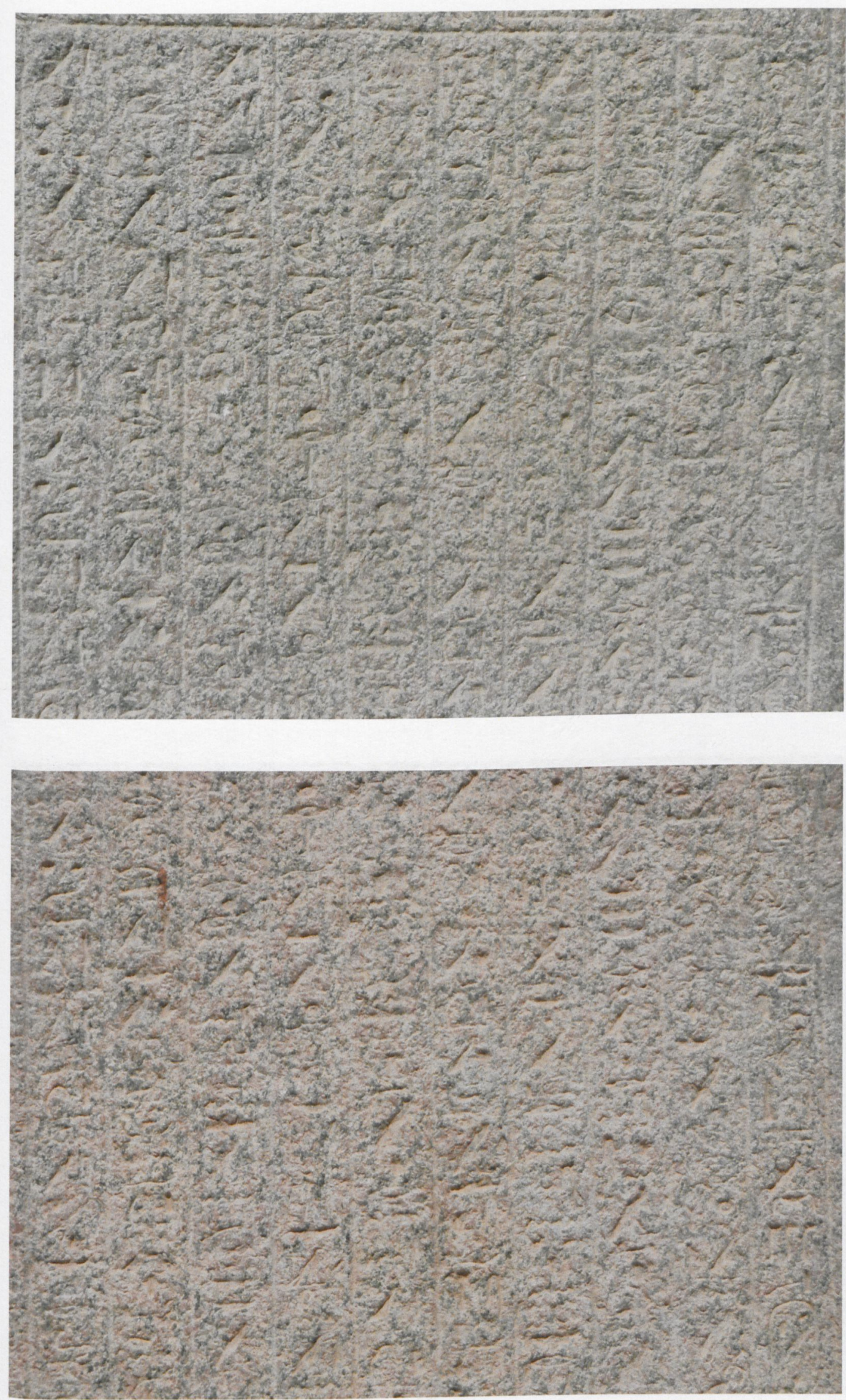

Abb. 2-3: Siegesstele des Amasis, rechts: 1. und 2. v. o., Kol. 1-10. 


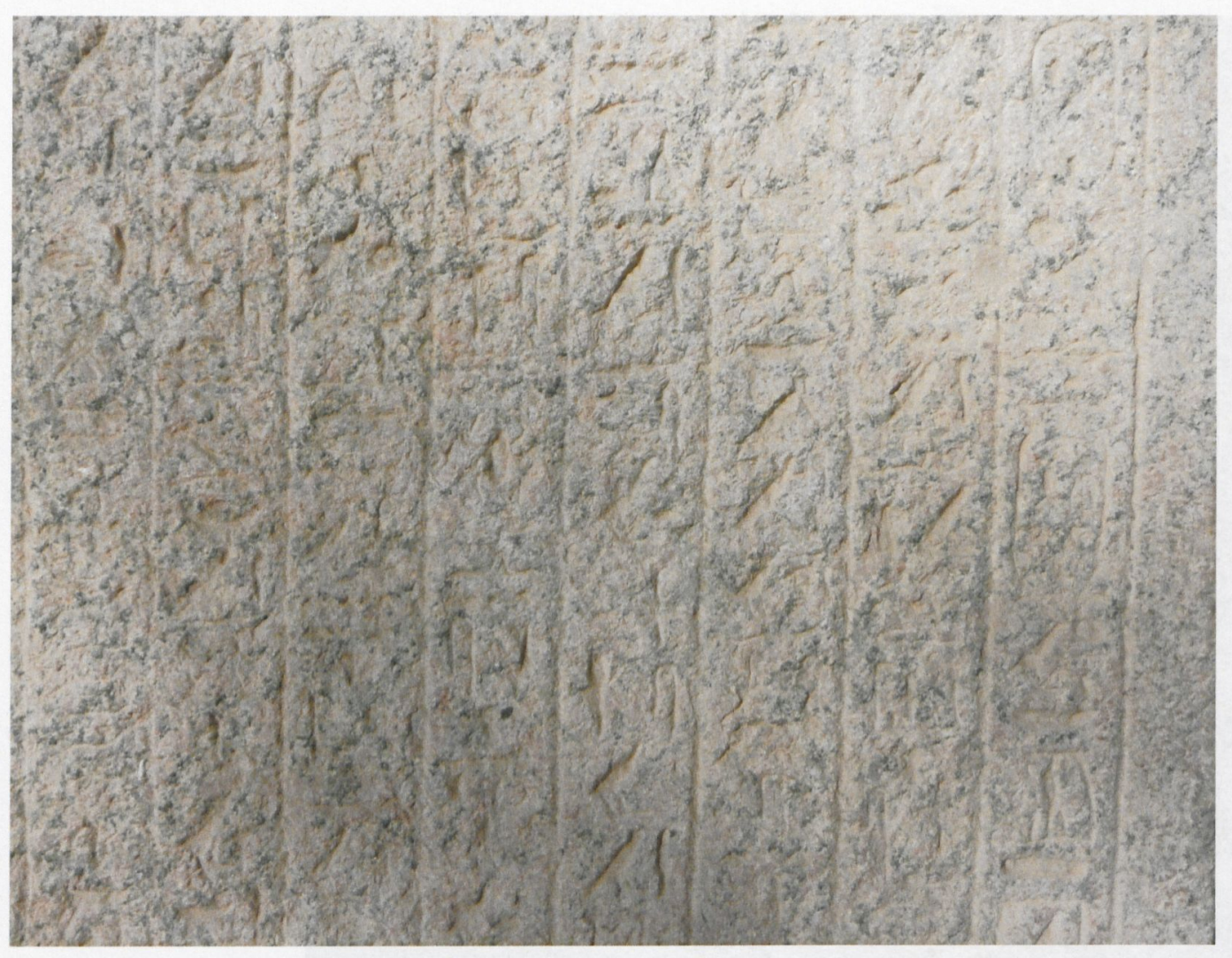

Abb. 4: Siegesstele des Amasis, rechts: 3. v. o., Kol. 1-8. 

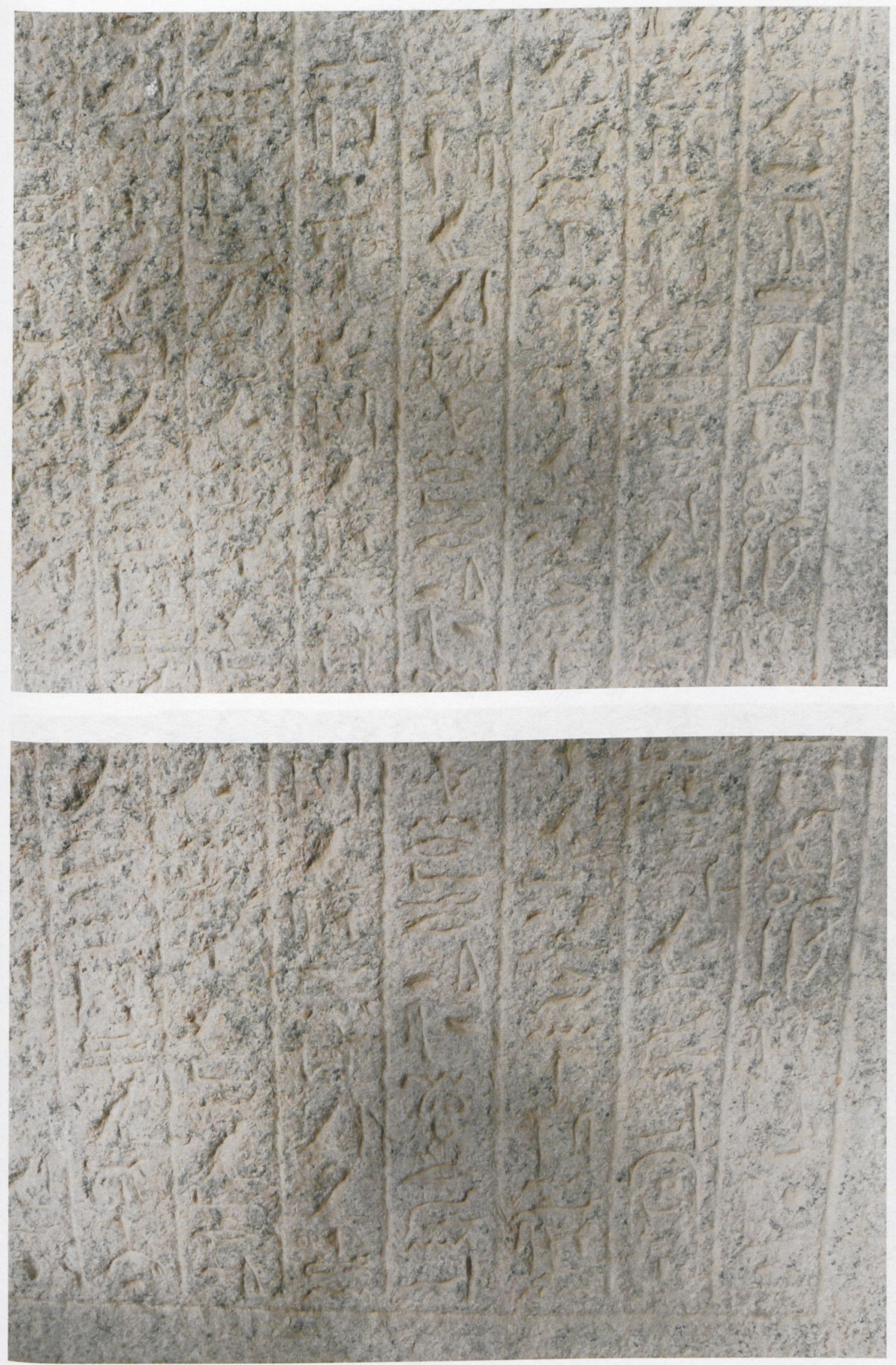

Abb. 5-6: Siegesstele des Amasis, rechts: 4. und 5. v. o., Kol. 1-7. 

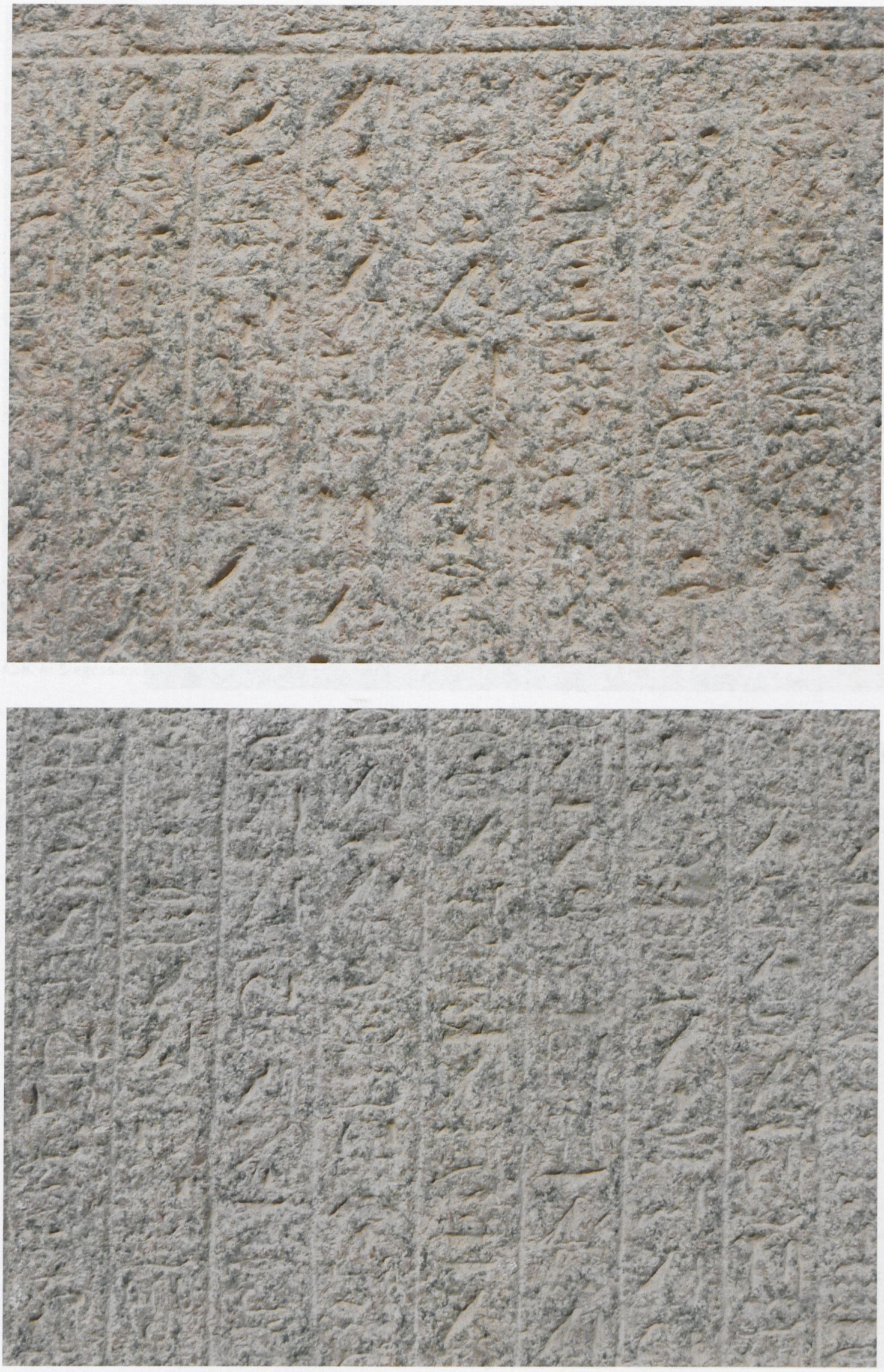

Abb. 7-8: Siegesstele des Amasis, Mitte: 1. v. 0.: Kol. 6-12 und 2. v. o.: Kol. 5-12. 

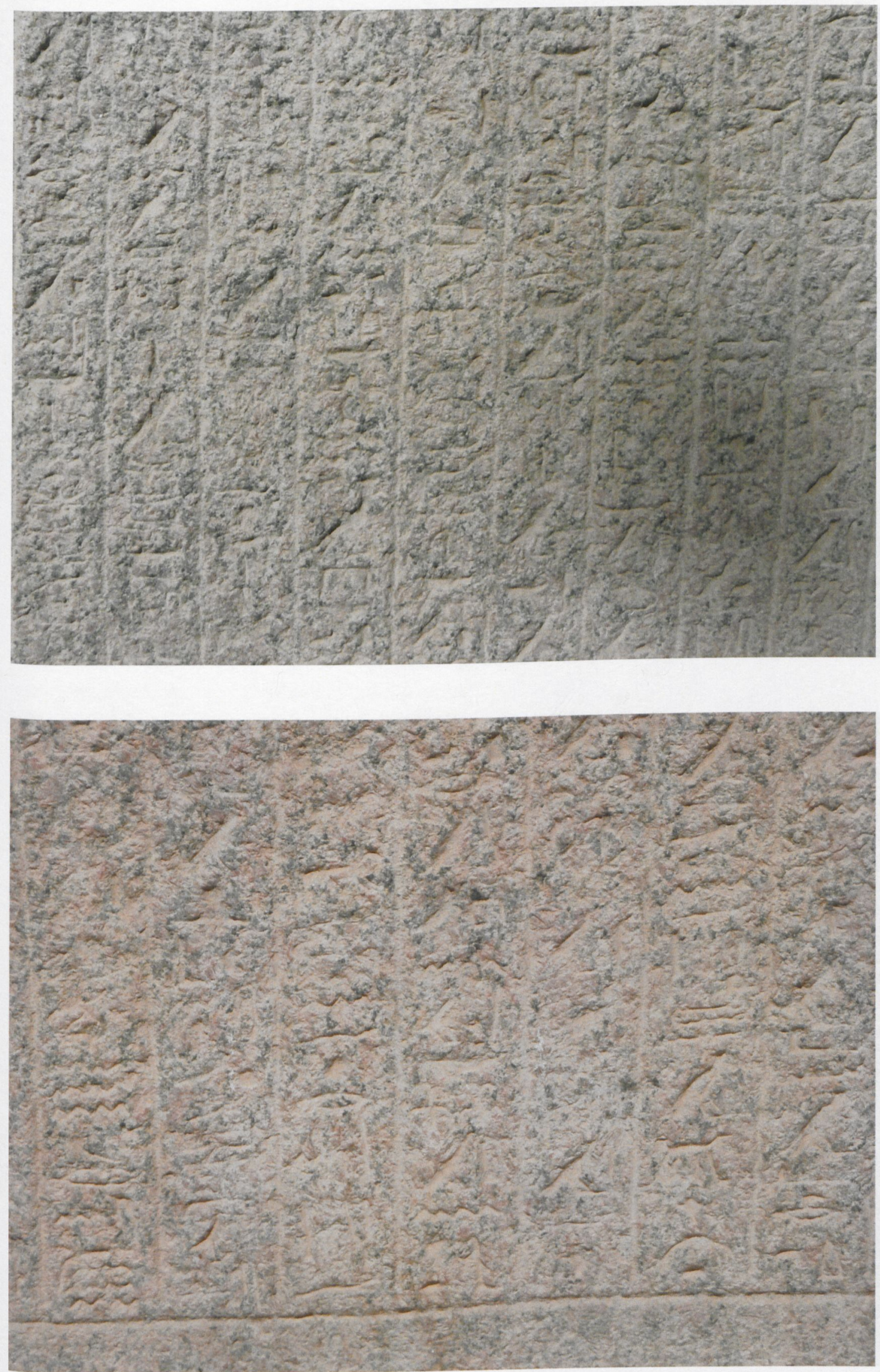

Abb. 9-10: Siegesstele des Amasis, Mitte: 3. v. o.: Kol. 4-12 und 4. v. o.: Kol. 6-12. 

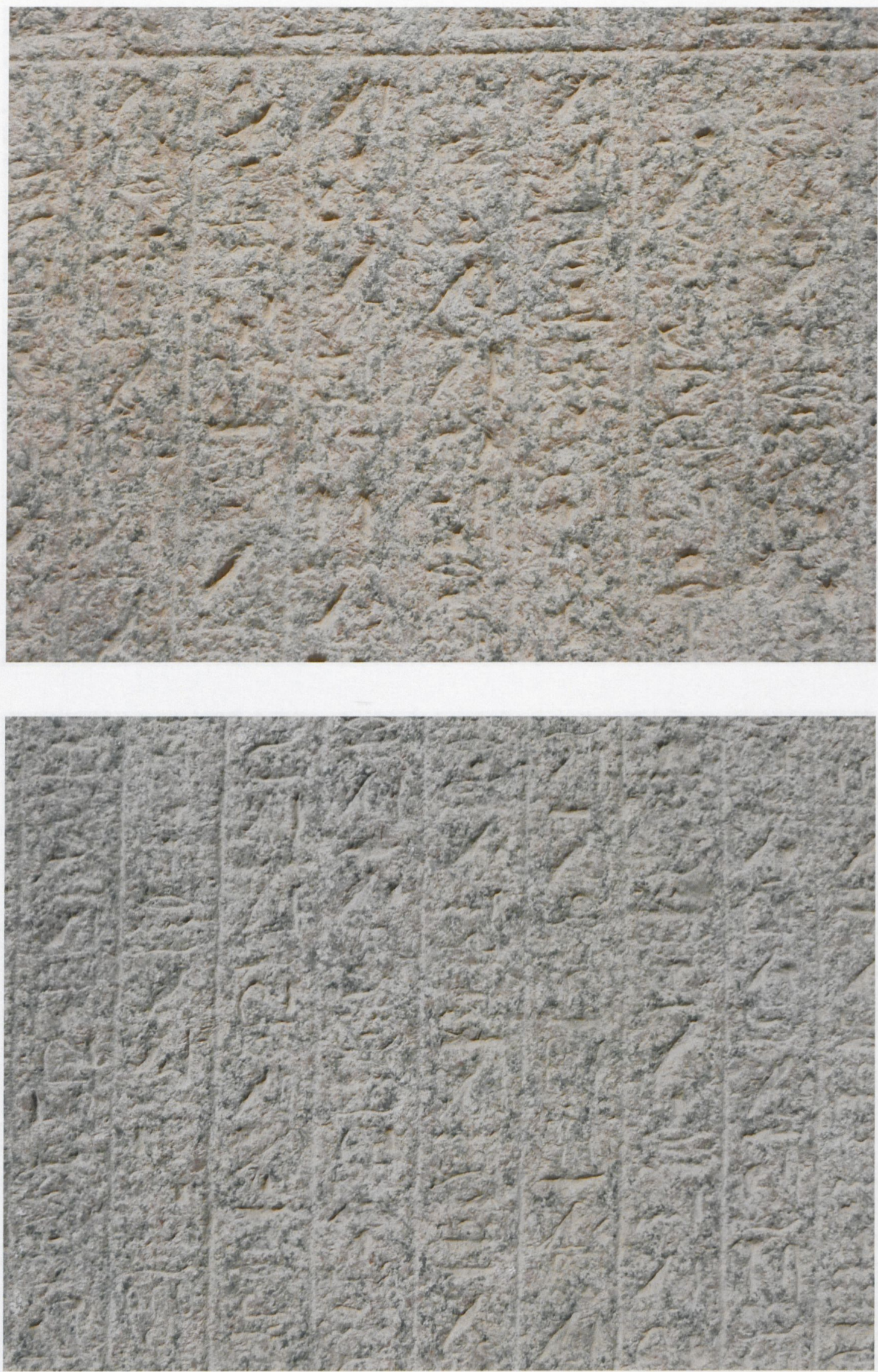

Abb. 11-12: Siegesstele des Amasis, links: 1. v. o.: Kol. 10-18 und 2. v. o.: Kol. 10-18. 

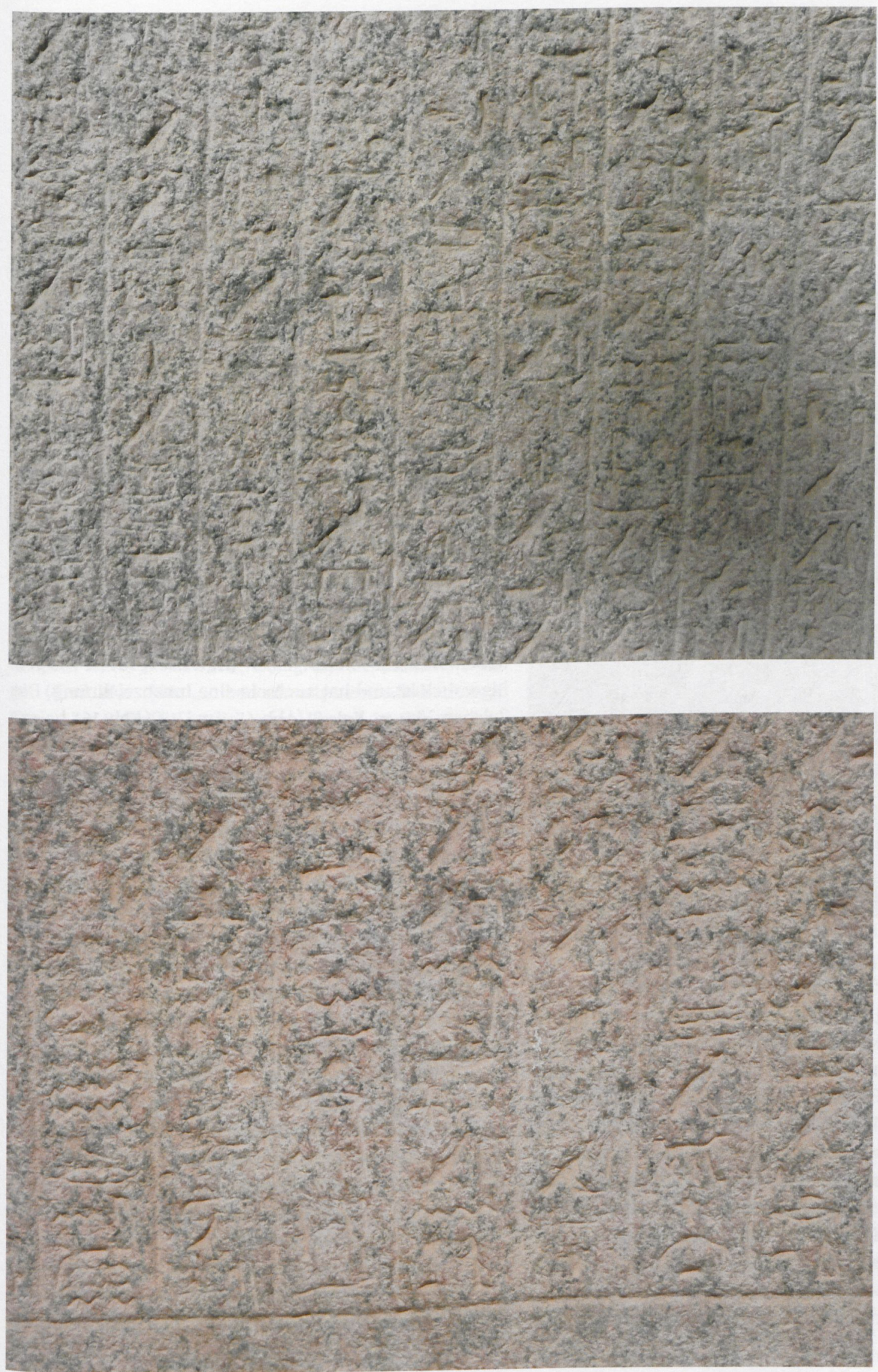

Abb. 13-14: Siegesstele des Amasis, links: 3. v. o.: Kol. 11-18 und 4. v. o.: Kol. 12-18. 
(60) Blöbaum, Herrscherlegitimation, 14 (= 135) liest hr $m$ f3j.sn jrj.n.f ,(er sah den Überheblichen) fallen in ihrem Erheben, das er gemacht hatte“. Ich kann von dem angeblichen $f j j$ keine Spur entdecken.

(61) Das $m h$-Zeichen am Beginn von Kol. 18 ist deutlich, das unklare Zeichen darunter lesen Daressy und Blöbaum $m$, aber es wäre ein Vogel ohne Beine. Es dürfte eher der wie in Kol. 7 stark gekrümmte $\underline{h}$ 3-Fisch sein, zu dem auch das Determinativ (sign-list Aa2, so auch Blöbaum) besser passt.

(62) So auch Blöbaum, Herrscherlegitimation, 14. Edel (GM 29, 17: „da bestattete er den Überheblichen ... an der Seite eines jeden rechtmäßigen Königs“, vielleicht von Herodot II,169 inspiriert), hatte Daressys Text vermutlich als $\langle r\rangle$ rmn (vgl. Wb II, 418,15) nswt $n b$ mnh verstanden. Statt $r m n$ ist aber ziemlich sicher $j r j n b$ zu lesen.

(63) Vor bwt ntrw muss ein Verb im sdm.n.f stehen. Das Zeichen vor $n . f$ ist fraglich, aber auf jeden Fall ein Vogel. Ich vermute $t^{3} j . n$. $f$ (also den $\underline{t} 3$-Vogel), aber der $z$-Vogel wäre auch nicht ausgeschlossen; dann müsste man htm.n.f „nachdem er vernichtet hatte“ lesen.

\section{Kommentar}

A. Die Inschrift des Amasis ist nach Funktion, Struktur und Inhalt eine klassische „Königsnovelle“, die zwei Episoden berichtet, die zeitlich deutlich voneinander getrennt sind, aber sachlich zusammengehören. Nicht so klassisch ist allerdings die Tatsache, dass hier ein Usurpator entscheidende Ereignisse seines Kampfes gegen den (zumindest der Erbfolge nach) legitimen Herrscher schildert und nicht einmal verschweigt, dass sein Gegner der legitime König ist.

Es wäre durchaus denkbar, dass Amasis auch schon nach der ersten Auseinandersetzung in seinem Jahr 1 propagandistische Inschriften anfertigen ließ, die dann, als Apries noch lebte und eine Gefahr darstellte, vermutlich weniger großmütig mit ihm umgingen. Sollte es sie jemals gegeben haben, wären sie aber zweifellos nach dem Krieg von Jahr 4 wieder entfernt worden. Tatsächlich vorhanden ist jedenfalls diese Siegesstele aus dem Jahr 4, und es ist sehr wohl möglich, dass es, nach Vorbild der Inschriften Psametiks II. zu seinem Nubienfeldzug (und denen des Taharka), mehrere gleiche oder ähnliche Exemplare gab, die in verschiedenen Tempeln aufgestellt waren ${ }^{9}$. Bei diesem Exemplar gibt es aller- dings eine Merkwürdigkeit: Aus den in Kol. 1 genannten Göttern, Chnum, dem „Herrn des Kataraktengebiets“, und Hathor von Biga(?), hat man auf eine Herkunft der Stele aus Elephantine geschlossen; daher ist sie heute im Nubischen Museum in Assuan. Auch das Material Rosengranit wäre bei einer solchen Herkunft besonders naheliegend. Andererseits ist die Stele in Kairo verbaut gefunden worden, das spricht eher für eine Herkunft aus Memphis. Eine sekundäre Verschleppung eines solchen Steins von Assuan nach Kairo wäre auch recht ungewöhnlich. Man könnte sich daher fragen, falls es wirklich mehrere Exemplare der Stele für verschiedene Tempel gegeben haben sollte, ob sie nicht sämtlich in Memphis ausgefertigt wurden und dann an die verschiedenen Tempel verteilt werden sollten. Man müsste dann annehmen, dass dieses Exemplar aus irgendeinem Grund nicht nach Süden weitergeleitet worden war.

B. Einer der strittigsten Punkte bei der bisherigen Behandlung der Inschrift war es, was mit der ominösen „Insel" in Kol. 3 und 13 gemeint ist. Wie oben (Anm. 6) dargelegt, ist aber mit Sicherheit nicht die Hieroglyphe für „Insel“ zu lesen, wie es Daressy und alle nach ihm getan haben. Das Zeichen für „Insel“ (sign-list N 18) ist viel weniger dick ist und hat auch nie eine Innenzeichnung. Die Zeichen hier in Kol. 3 (Abb. 15) und 13 (Abb. 16) haben aber zweifellos eine Innenzeichnung, und zwar mit größter Wahrscheinlichkeit das Auge (D 7), und damit ist $c_{n}(w)$ zu lesen. Ein Gewässername ${ }^{{ }^{n}} n w$ kommt auf der Naukratisstele in den Kolumnen $10(\vec{\circ})$ und $13(\overrightarrow{0})$ vor und bezeichnet dort den kanopischen Nilarm, s. Gauthier, DG I, 146; H. de Meulenaere, in: R. J. Demarée/ K. R. Veenhof, Zij Schreven Geschiedenis, Leiden, Löwen 2003, 430, n. 12; J. Yoyotte, in: F. Goddio/M. Clauss, Ägyptens versunkene Schätze, Ausstellungskatalog Berlin 2006, 319; A. S. von Bomhard, The Decree of Sais, Oxford 2012, 80 (g); vgl. auch F. Leclère, Les villes de Basse Egypte, BdÉ 144, 2008, 119. Damit ergibt sich nun eine ganz neue Situation für das Verständnis der Vorgänge im Jahr 1: Im ersten Teil der Stele, der vom Kampf mit den griechischen Söldnern des Apries berichtet, werden (außer der in Frage stehenden Bezeichnung) nur zwei andere Ortsnamen erwähnt, nämlich $J m 3 w$ (Kom el-Hisn) in Z. 11 als Ort, in dessen Nähe die Schlacht stattfand, und Sht-Mfk(3t) (vermutlich Terenuthis bzw. Kom Abu Billu ${ }^{10}$ ) in Z. 4, als der Ort, auf den der Angriff zielt. Edel (GM 29, 19) übersetzt „(sie nehmen ganz Ägypten,) sie erreichen Sht-mfk3t"; Leahy dagegen (JEA 74,190 ) fasst das Verb 


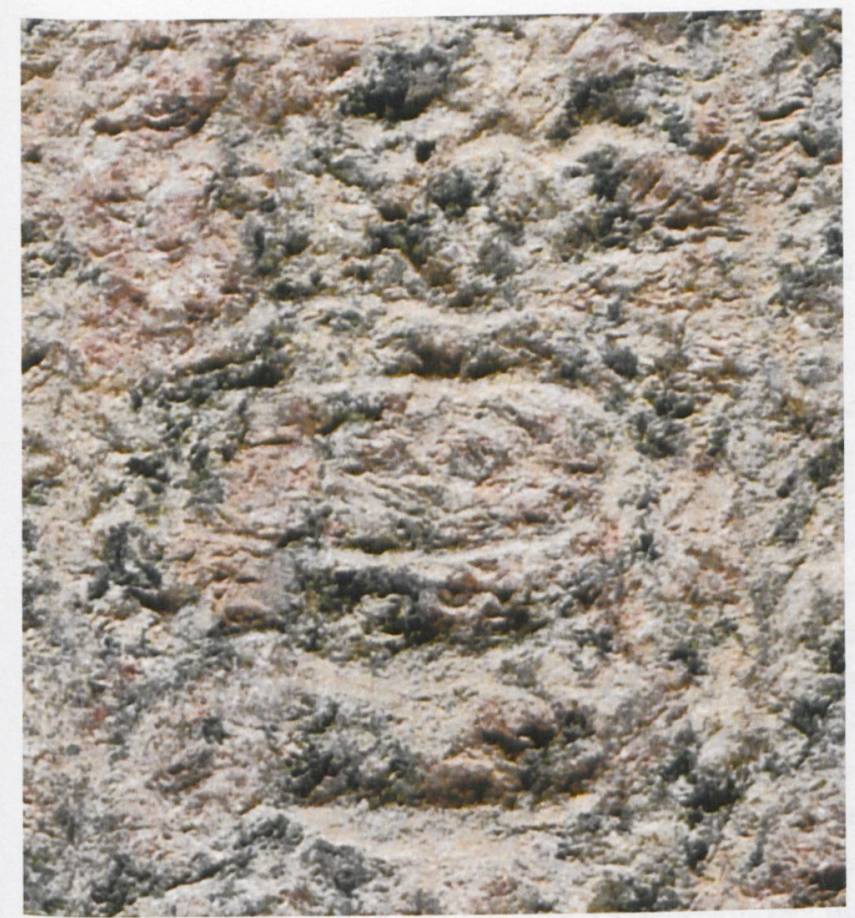

Abb. 15: Siegesstele des Amasis, Z. 3: ${ }^{\complement} n w$.

als präteritales $s \underline{d}$. $f$ auf (was grammatisch ohne weiteres möglich wäre) „... had reached Sht-mfk3t“, ebenso Spalinger (Civil War, 594: „... had already reached the site of Kom Abu Bellu“). Da die ganze Phrase im Zusammenhang aber lautet: jtj.sn Kmt $r$ 3w.s ph.sn Sht-mfk3t und die Feinde zu diesem Zeitpunkt unmöglich ganz Ägypten erobert haben können, sind die Verben sicher im optativisch/futurischen Sinne zu verstehen: „sie wollen/werden ganz Ägypten erobern und Sht-mfk3t erreichen“ oder auch, etwas freier: „sie drohen ganz Ägypten zu erobern und S. zu erreichen“. Sowohl Kom el-Hisn als auch Terenuthis lagen (nahe) am kanopischen Nilarm, ihre Erwähnung in der Stele ist vollkommen schlüssig, falls der Angriff über diesen Mündungsarm erfolgte. Offensichtlich nur aufgrund dieser Ortsnamen hatte schon Spalinger ganz selbstverständlich vorausgesetzt, dass die Schiffe des Apries auf diesem Nilarm fuhren (Civil War, 603 [15]: „He [Apries] traveled down the Canopic arm of the Nile and landed close to modern-day Kôm el-Hisn“; ibid., 594: „Apries ... had moved north along the western-most arm of the Nile"). Wieso er allerdings eine nördliche Richtung annimmt, ist mir rätselhaft ${ }^{11}$.

\footnotetext{
11 Ebenso nimmt Leahy, JEA 74, 192 oben an, dass Apries aus südlicher Richtung kam: „the stela places Amasis at Sais with his opponent moving north towards him“. Tatsächlich sagt die Stele nichts dergleichen. Ob die falsche Wiedergabe bei Daressy, Kol. 3 (hns $m$ $m h$, mit Stadtdeterminativ $=$ Norden) der Ausgangspunkt für diese These war? Die entsprechende Stelle war eigentlich schon von Edel
}

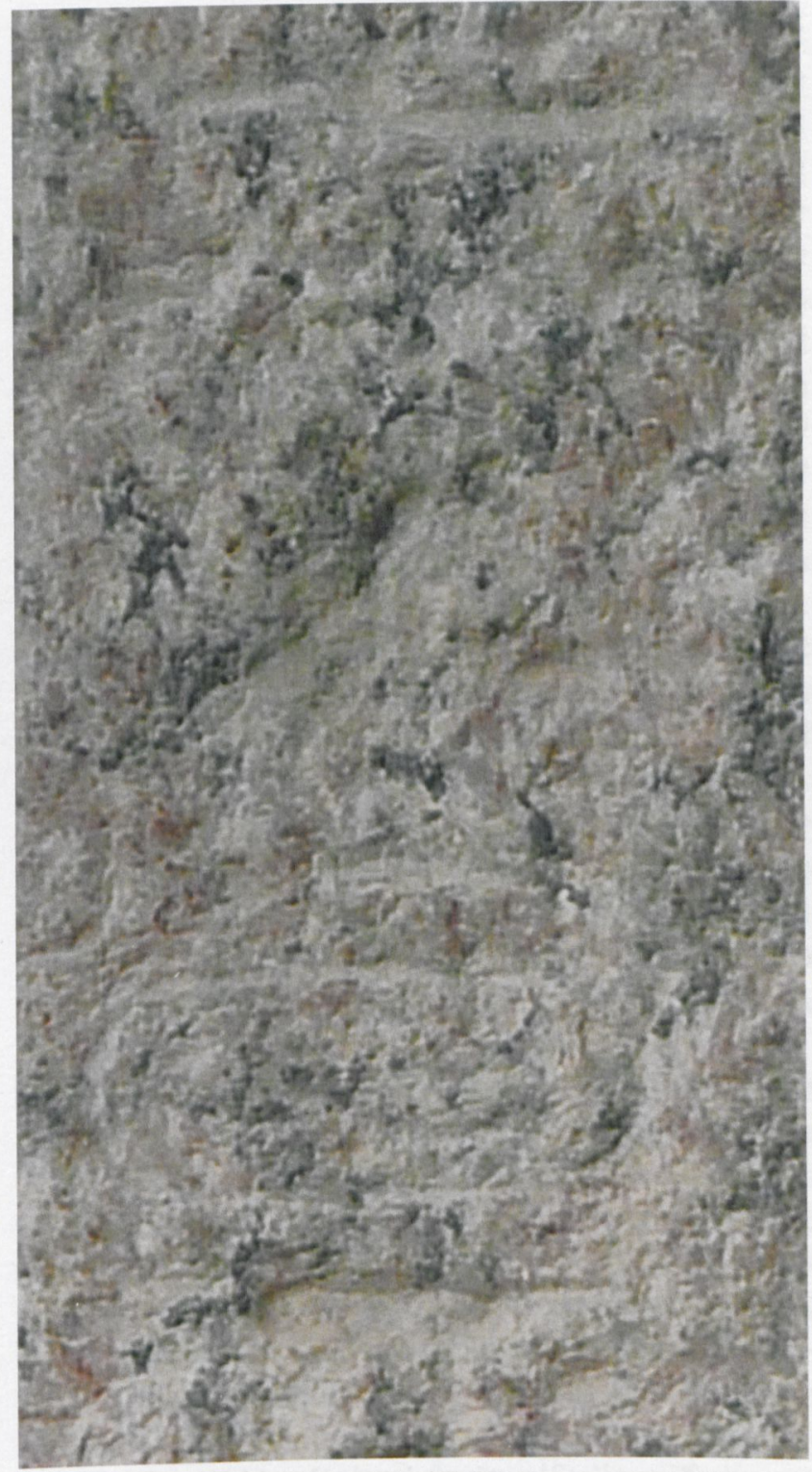

Abb. 16: Siegesstele des Amasis, Z. 13: ${ }^{c_{n}}$ w.

So, wie die Situation auf der Stele geschildert wird, war der Angriff offenbar eine Überraschung, und das kann er nur gewesen sein, wenn die Schiffe des Apries vom Meer her in den Nilarm einfuhren. Auch der Bericht der Boten in Kol. 2-4 ergibt auf diese Weise einen viel besseren Sinn: Man berichtet Amasis, wo der Feind ist und wohin er sich bewegt. In Edels Auffassung hätten die Boten dagegen erzählt, dass der Feind aus dem fernen Zypern kommt (woher sollten sie das wissen?), aber in keiner Weise, wo er aktuell steht. Allein dies ist schon unwahrscheinlich. Zudem wäre eine solche Bezeichnung

richtig wiedergegeben worden: „Ihre Herzen sind überheblich; sie sagen:" also jb.sn $m h \underline{h} r<\underline{d} d>$. Von einer Fahrt nach Norden ist keine Rede. 
für Zypern doch merkwürdig, wie Leahy ${ }^{12}$ völlig zu Recht sagte, „it [= „die Insel“ $]$ would be a curiously oblique reference to a place well known to Egyptians of the period“. Das gilt für seine eigene Idee, „die Insel“ als Bezeichnung für den Palast des Apries in Memphis zu verstehen, allerdings in noch stärkerem Maße. Zudem wäre die Formulierung, dass eine Festung (als Subjekt) „Schiffe fahre/rudere" so unidiomatisch, dass man es wohl ausschließen kann (s. o., Anm. 6 zur Übersetzung). Auch wäre es nur schwer vorstellbar, dass Apries mehr als ein halbes Jahr nach seinem Sturz eine große Armee bei Memphis zusammenziehen bzw. unterhalten konnte, ohne dass es schon vorher zu einer Entscheidung kam. Amasis hätte kaum abgewartet, bis diese Armee nach Norden angriff. Die Tatsache, dass Theben noch gut 8 Monate nach der Usurpation des Amasis nach Apries datierte, muss keineswegs bedeuten, dass Apries sich noch in Ägypten aufhielt ${ }^{13}$. Es war sicher bekannt, dass er Truppen gegen seinen Gegner zusammenzog, und man wird gerade in Theben eine endgültige Entscheidung abgewartet haben, da ja die Gottesgemahlin Anchnesneferibre, die nominelle Herrin der Thebais, eine Schwester des Apries war.

C. Geht man von einem Angriff auf dem kanopischen Nilarm aus, ergeben alle Quellen ${ }^{14}$ zusammengenommen am ehesten folgenden Ablauf:

1. Amasis wird vom Heer zum König erhoben, die Mission des Patarbemis scheitert (Hdt. II,162).

2. Apries, der ja schon ein Heer nach Kyrene entsandt hatte, wird sicher nicht sofort eine zweite Armee in der erforderlichen Stärke verfügbar gehabt haben. Aber es wäre durchaus vorstellbar, dass er diejenigen Truppen, die er gerade zur Hand hatte, dem Amasis entgegenschickte, denn zu diesem Zeitpunkt war ja noch nicht sicher, ob die Meuterer es wirklich auf einen bewaffneten Kampf gegen den legitimen König ankommen ließen, oder ob sie nur den Mund zu voll genommen hatten. Eine Schlacht bei Marea, wie sie Diodor $(I, 68)$ überliefert, wäre daher gar nicht so unwahrscheinlich, denn, wie Leahy zutreffend festgestellt hat, „Marea is a likely location for an encounter between a king based in Sais and an army returning from Cyrene“"15. Allerdings wäre dieses Treffen dann - gegen Diodor - nicht die entscheidende Schlacht

12 JEA 74, 194.

13 So Leahy, JEA 74, 195.

14 Leahy, JEA 74, 189.

15 JEA 74, 189. Vgl. auch W. Helck, Geschichte des Alten Ägypten. HdO I 1.3, Leiden, Köln 1968, 255. des Amasis gegen die 30000 Söldner des Apries gewesen, sondern wohl eher ein Gefecht in kleinerem Rahmen, das aber Apries zur Flucht aus Sais zwang.

3. Apries müsste sich dann in einen Ort zurückgezogen haben, der es ihm gestattete, seine Truppen zu sammeln und neue $\mathrm{zu}$ werben, vielleicht am ehesten in die Lager der Söldner bei Tell Defenneh ${ }^{16}$. Amasis wird wohl gleichfalls versucht haben, seine Heeresmacht zu vergrößern, und die ägyptischen Kerngebiete zumindest in Unter- und Mittelägypten unter seine Kontrolle zu bringen. Die Stele sagt expressis verbis, dass Amasis in Sais residierte. Auch wenn man diese Angabe bloß dem Texttyp „Könignovelle“ zuschreiben wollte ${ }^{17}$, die recht konkrete Behauptung in Kol. 11, dass der König „auf Befehl der Neith“ einen Bogen aus Silber (oder Gold) mitführte, dürfte ebenfalls darauf deuten, dass Amasis schon in Sais residierte (s. auch unten).

4. Von Tell Defenneh aus hätte sich wohl ein überraschender Angriff zur See und in den kanopischen Nilarm organisieren lassen, um Amasis in Sais zu treffen. Der auf der Stele genannte Ort $J m 3 w$ (Kom elHisn), vermutlich identisch mit Herodots Schlachtort Momemphis, wäre bei dieser Lokalisierung der gegnerischen Parteien naheliegend.

D. Der kanopische Nilarm wird nach der Schlacht „auf all seinen Seiten abgedämmt", sicher nicht gegen Wasser, sondern gegen Feinde. Die Stellung der letzteren Episode zwischen Schlachtgeschehen und anschließendem Gottesopfer legt nahe, dass es sich um eine kurzfristig eingeleitete Blockade des Nilarms handelte, der möglicherweise nachdrängende Feinde abwehren sollte, also in gewissem Sinne noch zum Schlachtgeschehen zählte. Es wäre aber immerhin denkbar, dass aufgrund der Geschehnisse gerade dieser Nilarm, von dem aus ja auch die Residenzstadt Sais leicht erreichbar war, auch längerfristig besonders gut gesichert wurde. Und das könnte eine der Erklärungen dafür sein, dass ab der Zeit des Amasis ${ }^{18}$ alle fremden Schiffe nur in den Nilarm von Kanopos einfahren durften.

E. Apries, der Vorgänger und Gegner des Amasis, wird auf der Stele mehrfach erwähnt:

- Eindeutig ist die Erwähnung am Ende von Kolumne 2: Der Bote nennt als Auftraggeber der Invasion schlicht „Apries“, in Kartusche geschrieben, aber

16 Vgl. auch Leahy, JEA 74, 195-6.

17 Vgl. etwa Lloyd, Herodotus, III, 178-9.

18 Vgl. Lloyd, Herodotus, III, 229-30. 
ohne Titel. Eine Umschreibung wäre hier wenig sinnvoll, er muss ja klar melden, von wem die Rede ist. Ein ägyptischer Königstitel (der immer nur einer lebenden Person zukommt) wäre andererseits unangemessen, weil er zu dem neuen König Amasis spricht.

- Am Ende von Kol. 7 wird, als Gegensatz zu dem von der Maat geleiteten Amasis, ein „Wüterich“ genannt, der „wie der Hund an der Leiche gehandelt hat“. Damit kann wohl nur Apries gemeint sein ${ }^{19}$. Der „Wütende“ (3d) kommt in der ägyptischen Phraseologie gewöhnlich als Objekt zum Verb hsf „abwehren“ vor $^{20}$, und auch hier ist offensichtlich derjenige gemeint, den man nun abwehren muss. Es ist sicher bezeichnend, dass diese abwertende Metapher (und der anschließende schimpfliche Vergleich mit einem Hund) gerade in der Rede der Höflinge und beflissenen Anhänger des Amasis erscheint. Er selbst dagegen erwähnt zunächst (im Jahr 1) seinen Gegner gar nicht.

- Am Ende von Kol. 10 wird berichtet (keine wörtliche Rede), dass Amasis die zu seiner Armee gehörigen Pferde selbst ausgewählt hatte „unter der Majestät des" [Satzende]. Hier ist offenbar ganz bewusst der Name des Apries ausgelassen worden. Es wäre leicht möglich gewesen, das $h r h m n$ durch ein unverfängliches Zeitadverb wie „vorher“ oder „früher“ zu ersetzen $^{21}$. Wieso man diese seltsame Ausdrucksweise wählte, ist mir rätselhaft.

- In Kol. 16 erwähnt Amasis in seiner Rede (in leider unklarem Zusammenhang) einen „Frevler, der keinen Namen hat“. Auch hier kann nur Apries gemeint sein, dessen Freveltat zweifellos darin gesehen wird, dass er mithilfe ausländischer Feinde Ägypten angreift.

- In Z. 17 und 18 schließlich wird der tote Apries als $m h$-jb.f bezeichnet. Edel hatte dies ohne weitere Erläuterung mit „der Überhebliche“ wiedergegeben ${ }^{22}$ und Blöbaum hat sich dem angeschlossen ${ }^{23}$. Auf den ersten Blick wirkt das etwas befremdlich, denn normalerweise hat dieser Ausdruck ja eine durchaus positive Bedeutung, oft im Zusammenhang von König

19 So schon Breasted, AR IV, § 1004.

$20 \mathrm{~Wb}$ I, 24, 22.

21 Wenn dagegen aus irgendeinem Grund ausgedrückt werden sollte, dass Amasis im Dienst seines Vorgängers gewesen war, hätte man eine Formulierung wählen können wie etwa auf der Stele Nektanebos' I. (der gleichfalls durch einen Putsch an die Macht gekommen war) aus Hermopolis: „(in der Zeit) des Königs, der vor ihm gewesen war“ (wn $\underline{h r}-\underline{h t} t . f)$, s. Roeder, ASAE 52, 1954, 389.

22 Edel, GM 29, 17; vgl. auch ibid., 18.

23 Blöbaum, Herrscherlegitimation, 164-5. und Gefolgsmann (,sein Vertrauter“ u. ä.) ${ }^{24}$. In diesen (üblichen) Fällen ist es allerdings so, dass eine Person A (z. B. der Funktionär) das Herz einer zweiten Person B (z. B. des Königs) „füllt" und dadurch sein Vertrauen hat. Hier ist aber wohl gemeint, dass Apries sein eigenes Herz „füllt“, was durchaus eine andere Bedeutung haben könnte, wie etwa ,der Überhebliche“ oder „der Optimist“ (im ironischen Sinne). Für diese Bedeutung kann man darauf verweisen ${ }^{25}$, dass auch von den Söldnern des Apries in Kol. 3 gesagt wird „ihre Herzen sind gefüllt [zuversichtlich]“ (jb.sn $\mathrm{mh}$ ) und die Asiaten in Z. 14 rebellieren $\mathrm{m} \mathrm{mh}$ $j b . s n$,in ihrer Zuversicht/Überheblichkeit“. In beiden Fällen „füllen“ diese Personen ihr eigenes Herz. Zumindest erwägen könnte man aber auch eine andere Lösung: Da in beiden Fällen, wo Apries als $m h-j b . f$ bezeichnet wird, ein Suffix vorausgeht, das sich auf Amasis bezieht („er [Amasis] sah den $m h-j b . f^{*}$ bzw. „er [Amasis] bestattete den $\left.m h-j b . f^{\prime \prime}\right)$, könnte auch das Suffix von $m h-j b . f$ auf Amasis verweisen. Dann sollte es vielleicht so etwas wie „den er bemitleidet“ bedeuten. Das ist aber zweifellos die sehr viel unwahrscheinlichere Möglichkeit.

In jedem Fall ist es ersichtlich, dass man bei der Konzeption des Stelentextes die Frage der Bezeichnung des entthronten Königs sehr differenziert gehandhabt hat.

F. Dagegen werden die Truppen Nebukadrezars und ihr Vorgehen nur in allgemein gehaltenen Ausdrücken geschildert, sie stehen ersichtlich nicht im Zentrum der Darstellung, obwohl sie ja die eigentlichen Angreifer sind; für sie dürfte Apries kaum mehr als eine Galionsfigur gewesen sein. Nur aus einer einzigen Stelle erfährt man, wer eigentlich die Angreifer sind: In Z. 14 wird dem König gemeldet, dass die „Asiaten sich empört haben“ und Ägypten zu Lande und zu Wasser ${ }^{26}$ angreifen. Dann wird sehr knapp berichtet, wie die Feinde durch ein (von Gott gesandtes) Unwetter und durch die eigenen Truppen besiegt worden sind, und gleich darauf wendet der König seine Aufmerksamkeit wieder dem gefallenen Apries zu und lässt ihn bestatten. Der babylonische Invasionsversuch dient in dieser Darstellung nur mehr als Hintergrund für die Auseinandersetzung zwischen Amasis und Apries.

\section{Wb II, 118.}

25 Vgl. auch Blöbaum, Herrscherlegitimation, 165.

26 Aus dem einzigen Satz dazu ( $n$ tjw $m h w^{c} w$ fjj.sn tijw) wird nicht klar, ob die Schiffe der Gegner auf dem Meer oder auf einem Nilarm „segeln“. 
Im Übrigen ist es eher unwahrscheinlich, dass Apries tatsächlich in der Schlacht gefallen oder ertrunken ist, da sich Könige nur selten solch einem Risiko aussetzen. Näher liegt wohl, dass er gefangen oder von den Truppen der Babylonier nach der Niederlage ausgeliefert wurde. Die Version bei Herodot, dass er nach kurzer Gefangenschaft getötet wurde, könnte durchaus zutreffen.

G. Die Angabe in Kol. 10, dass Amasis, bevor er selbst König wurde, Pferde für die Armee ausgesucht habe, könnte einen Hinweis auf seine Stellung unter Apries geben. Es wird oft gesagt, dass er „General“ war, und manchmal wird er sogar konkret mit dem aus dem $\mathrm{Nu}-$ bienfeldzug Psametiks II. bekannten General $J c h$-msw identifiziert $^{27}$. Das ist schon aus chronologischen Gründen mehr als unwahrscheinlich. Immerhin sollte er aufgrund der Tatsache, dass er zu einer meuternden Armee geschickt wurde, auf jeden Fall etwas mit Militär und Soldaten zu tun gehabt haben. Es wäre also zu erwägen, ob er nicht unter Apries für die mit Pferden ausgerüsteten Truppen (ob nun „Kavallerie“ oder Streitwagen) zuständig, also eine Art „Marschall“ war. Nach Herodot sitzt er zu Pferde, als der königliche Gesandte Patarbemis auf ihn trifft. Und es wäre ja möglich, dass Amasis nicht zuletzt deshalb als Unterhändler ausgesucht worden war, weil er zu Pferd die meuternde Armee schnell erreichen konnte.

H. Auf der Siegesstele des Amasis spielen natürlich auch die Götter eine Rolle. Jeweils nach der gewonnenen Schlacht werden ihnen Opfer dargebracht, aber beide Fälle werden unterschiedlich behandelt: Nach der Schlacht im Jahr 4 stiftete Amasis dauerhaft (smn.n.f) ein sehr umfangreiches Opfer ( $\left.h t p-n t \underline{t} c_{S}^{2} 3 w r t\right)$ für die Götter von Ober- und Unterägypten (d. h. wohl für alle wichtigeren Götter Ägyptens). Nach dem Sieg im Jahr 1 begeben sich Leute „ohne Ende“ zum Gott (ntr, im Singular) und bringen Opfergaben zu ihm. Es wird ausdrücklich gesagt, dass der Gott „vernachlässigt“ war und sein Opfer „gesucht" habe. Das wird mit der (leider etwas schwierig zu verstehenden) Angabe in Kol. 3-4 zu verbinden sein, dass die einfallenden Söldner des Apries auch den Unterhalt eines Gottes nicht verschonten. Es fragt sich, wie das konkret gemeint ist. Wenn es sich um Besetzung und Plünderung von Tempeln handelte, würde man sich nicht so gewunden ausdrücken, und am Unterlauf des westlichsten Nilarms dürfte es auch kaum größere Tem-

27 Bspw. N. Grimal, Histoire de l'Egypte ancienne, Paris 1988, 437; I. Shaw/P. Nicholson, The British Museum Dictionary of Ancient Egypt, London 1995, 19 und sonst öfter. pel gegeben haben. Vielleicht ist eher gemeint, dass die Invasoren ihre Versorgung auch aus Scheunen oder von Feldern bestritten, die zum Landbesitz von Tempeln gehörten ${ }^{28}$. Sowohl der geschädigte Gott in Kol. 3 als auch der Gott in Kol. 13, zu dem man nach dem Sieg geht, werden nicht weiter spezifiziert, es handelt sich (grammatisch) wohl um einen generellen Singular. Inhaltlich dürfte die nicht weiter spezifizierte Bezeichnung am ehesten so zu erklären sein, dass mehrere Götter geschädigt worden waren (und später wieder ihre Opfer bekamen). Auf jeden Fall sollen die beiden Passagen (Kol. 3-4; 13) wohl vor allem deutlich machen, dass Apries in seinem Kampf keine Rücksicht auf die Götter nahm.

In Kol. 11 gibt es den einzigen Fall auf dieser Stele, wo (abgesehen von der Königstitulatur in Kol. 1) eine konkrete Gottheit genannt wird: Amasis führt in der Schlacht einen Prunkbogen mit sich, der ,auf Befehl der Neith" mit seinem Namen versehen ist. Das erinnert ein wenig an die Szenen und Textpassagen, wo Amun oder ein anderer Gott dem König ein Schwert überreicht ${ }^{29}$. Bei der Kriegsgöttin Neith ist es dagegen nur angemessen, dass sie ihm einen Bogen, ihr eigenes Abzeichen ${ }^{30}$, anbefiehlt. Auf der Statue Darius' I. aus Susa wird vom König gesagt: „Neith hat ihm ihren Bogen gegeben, der in ihrer Hand ist, um all seine Feinde niederzuwerfen ( $r d j$ n.f Njtt

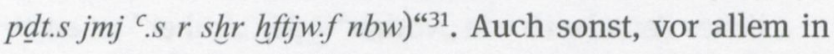
späten Tempelinschriften, sind entsprechende Aussagen belegt, z. B. dj.j n.k jwnt.j $r d r$ btnw.k ,ich gebe dir [dem König Ptolemaios VI.] meinen Bogen, um zu vertreiben deine Widersacher" ${ }^{* 32}$, und die gleiche Phrase kommt auch mit dem šmrt-Bogen vor (dj.j n.k šmrt.j $r d r$ btnw.k $)^{33}$,

28 Posener, Revue de philologie, de littérature et d'histoire ancienne 21, 1947, 121-30 hatte tatsächlich vermutet, die Angabe auf der Statue des $N h t-H r-h b$ (jetzt Berlin $1048+3 / 98$ ), dass die Opfergaben der Götter nicht mehr ,aus diesem Ort“ geliefert würden (s. Tresson, Kêmi 4, 1933, 130, Z. 15-6; pl. IX), könne sich auf die Situation am Beginn der Usurpation des Amasis beziehen (,la flotte grecque parcourt le Delta, l'activité des partisans d'Apriès peut gêner les transports et provoquer une interruption dans la livraison des offrandes“, ibid., 129).

29 Bspw. Israelstele, Z. 15 (KRI IV, 16,10) oder in der großen Inschrift des Merenptah in Karnak (Z. 29), wo ihm Ptah im Traum erscheint und ein Schwert überreicht (KRI IV, 5,12), vgl. auch Th. von der Way, Göttergericht und „Heiliger“ Krieg im alten Ägypten. SAGA 4, Heidelberg 1992, 41; C. Manassa, The Great Karnak Inscription of Merneptah. YES 5, New Haven 2003, 117-22; Schulman, in: B. Bryan/D. Lorton, Essays in Egyptology in Honor of Hans Goedicke, San Antonio 1994, 265-95.

30 Vgl. auch die Beiworte in LGG VIII, 270.

31 Yoyotte, Cahiers de la Délégation Archéologique Française en Iran 4, 1974, 182; 213; 255.

32 Esna II, 33 (14), Z. 14 (Rede der Neith).

33 Ibid., 49 (18), Z. 12. 
der auf der Stele des Amasis genannt wird. Ob die göttliche Ermächtigung des Amasis, einen solchen Bogen zu führen, allerdings eine konkrete Zeremonie voraussetzt, z. B. im Rahmen eines Orakels, ist schwer zu sagen.

Auf jeden Fall stützt diese Passage die Annahme, dass Amasis schon vor dieser Schlacht im Besitz von Sais war (s. o.). Sofern er Sais noch gar nicht eingenommen hatte, wäre eine solche Lüge für die Zeitgenossen deutlich erkennbar gewesen und vermutlich als gotteslästerlich empfunden worden.
In Kol. 16 schließlich ist es wiederum nur allgemein der „Gott“, an den sich Amasis wendet: ${ }^{c} h^{c} h m . f h r{ }^{c_{S}} m n$ $n t r$ „seine Majestät stand beim Kultbild des Gottes“. Diese Handlung des Amasis fand vermutlich unmittelbar vor der Schlacht statt. Die Stele sagt nichts über den Ort der Schlacht, außer dass die Feinde auf dem „Horusweg“ heranrücken. Sie dürfte daher, wie Leahy sicher zu Recht vermutet ${ }^{34}$, „probably near the eastern frontier“ ausgetragen worden sein. Dementsprechend müsste es dann um einen der im östlichen Delta verehrten Göttér handeln, dessen genaue Identität für den Zweck der Inschrift unerheblich war. 